\title{
UAV-aided Wireless Power Transfer and Data Collection in Rician Fading
}

\author{
Yuan Liu, Student Member, IEEE, Ke Xiong, Yang Lu, Member, IEEE, Qiang Ni, Senior Member, IEEE, \\ Pingyi Fan, Senior Member, IEEE, Khaled Ben Letaief, Fellow, IEEE
}

\begin{abstract}
A UAV-aided wireless power transfer and data collection network is studied, where it is assumed that when the harvested energy at the sensor node ( $\mathrm{SN}$ ) cannot surpass its circuit activation threshold or the received data rate at UAV falls below a minimal required rate threshold, the information outage occurs. The closed-form expressions of energy outage probability and rate outage probability are derived at first, and then the overall outage probability and coverage performance of the system are analyzed. Based on which, an optimization problem is formulated to minimize the overall outage probability by optimizing UAV's elevation angle and the time splitting (TS) factor. Since the problem is non-convex and has no known solution, an alternating optimization (AO)-based algorithm with Golden-section (GS) based linear search method is designed to find the global optimal solution. In order to explore the maximum coverage area of the UAV for a given tolerable outage probability, another optimization problem is also formulated to maximize the coverage range by optimizing UAV's elevation angle. By using Karush-Kuhn-Tucker (KKT) conditions, the closed-form solution of the optimal elevation angle for maximizing the coverage area is derived. Monte Carlo simulations verify the accuracy of the derived closed-form expression of the overall outage probability and the semi-closed-form expressions of the optimum UAV's elevation angle and TS factor. It shows that there exist a unique optimum elevation angle and the TS factor to achieve the minimum overall outage probability, and significant performance gain can be obtained by using our proposed optimization scheme. The developed theoretical results can be useful to the design of UAV-aided wireless communication systems with wireless power transfer.
\end{abstract}

Index Terms-UAV communication, wireless power transfer, data collection, Rician fading, outage analysis.

This work was supported in part by the National Natural Science Foundation of China (NSFC) under Grant no. 62071033, by the National Key R\&D Program of China under Grant no. 2020YFB1806903, by the Fundamental Research Funds for the Central Universities under Grant no. 2020JBZD010, and also by the Fundamental Research Funds for the Central Universities under Grant no. K20RC00020 and no. 2020YJS023. (Corresponding author: Ke Xiong.)

Y. Liu, K. Xiong and Y. Lu are with the School of Computer and Information Technology, Beijing Jiaotong University, and also with the Beijing Key Laboratory of Traffic Data Analysis and Mining, Beijing Jiaotong University, Beijing 100044, China (e-mail: liuyuan@bjtu.edu.cn; kxiong@bjtu.edu.cn; yanglu@bjtu.edu.cn).

Q. Ni is with the School of Computing and Communications and Data Science Institute, Lancaster University, UK (e-mail: q.ni@lancaster.ac.uk).

P. Y. Fan is with the Beijing National Research Center for Information Science and Technology, Tsinghua University, Beijing 100084, and also with the Department of Electronic Engineering, Tsinghua University, Beijing 100084, China (e-mail: fpy@tsinghua.edu.cn).

K. B. Letaief is with the Department of Electrical and Computer Engineering, Hong Kong University of Science and Technology (HKUST), Hong Kong, and also with the Pengcheng Laboratory, Shenzhen, Guangdong 518055, China (e-mail: eekhaled@ust.hk).

\section{INTRODUCTION}

\section{A. Background}

Recent advancements in Internet of Things (IoTs) and 5G/B5G have aroused numerous applications, including weather monitoring, intelligent transportation, smart agriculture, emergency search and rescue [四] - [䧃]. In these emerging intelligent applications, a large number of sensor nodes (SNs) are deployed in IoTs to collect environmental data and then upload data to upper-layer servers for computing and decisionmaking. As the SNs are usually powered by small-size batteries with limited energy storage capacity, they are required to be replaced or recharged up periodically [ $[$ ]. To release the labor cost and risk of manual battery replacement, by powering low-power SNs in a self-sustainable way, radio-frequency (RF) signal based wireless power transfer (WPT) has been widely regarded as a promising solution [回]- [8], as RF-based WPT is controllable, relatively reliable and capable of transferring power at a distance of up to tens of meters [Q].

Traditionally, dedicated RF energy transmitters (ETs) and data collectors are usually deployed at fixed locations. To cover low-power SNs in large-scale IoTs, it requires to deploy massive ETs and data collectors, which yields high deployment cost, thus hindering the large-scale deployment of WPT networks [एँ]]. When the transmitted RF signals propagate over wireless links, they may be significantly attenuated by channel shadowing and fading, so both the wireless energy transfer efficiency and the information delivery efficiency are deteriorated [प]]. As a result, the SNs located far away from the ETs and data collectors may not be well served due to the weak wireless links.

Recently, unmanned aerial vehicles (UAVs) have emerged to act as aerial base station (BS) for emergency network response, fast communication service recovery and ubiquitous coverage, especially in remote areas with insufficient terrestrial infrastructures [四]- [四]. UAV can also act as a mobile relay to help forward information for SNs []], a mobile charger to wirelessly charge IoT SNs [[13], or an aerial data collector to gather data from IoT SNs [14]]. Particularly, the line-of-sight (LoS) channels enable UAVs to wirelessly cover SNs much better than terrestrial communications [[5]], which expand the services scope and reduce the system deployment cost of IoT systems outdoors. Moreover, the controllable mobility enables UAVs to fly closer to SNs for establishing strong communication links, which greatly enhances the WPT efficiency for charging SNs, and also saves energy of SNs for uploading data to UAVs, thus prolonging the network lifetime of IoTs. 


\section{B. Related Work}

Owing to UAVs' merits such as on-demand operations, flexible deployment, controllable mobility, and superior link quality, UAV-aided wireless power networks have attracted

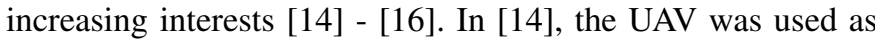
a relay to assist the users in computing or further offloading tasks to the BS for computing, where the weighted sum energy consumption of the UAV and users was minimized. In [L5], a legitimate UAV was exploited to process computing tasks for users in the presence of multiple eavesdropping UAVs, where the minimum secrecy capacity was maximized. In [ए6], the UAV was deployed with WPT to power SNs, where the sum energy received by all energy receivers was maximized. However, in these works, only the free-space path loss of the channels was considered, which ignored the effects of stochastic fading including the multipath fading.

As the channel between a UAV and a SN also suffers multipath fading caused by reflection, scattering, and diffraction by the ground obstacles such as bumpy ground, grass, trees and buildings, some recent works have begun to discuss the performance of UAV-aided wireless networks in fading channels [[7]]- [24]. In [ए]], the system outage probability was minimized in Rayleigh fading, with UAV acting as a relay. In [एष], the coverage probability was analyzed in Rayleigh fading, where a single UAV acted as a mobile user to connect with a ground BS. As Rayleigh fading model is only applicable to the cases when there is no LoS link from the transmitter to the receiver, and the air-to-ground (A2G) channels are often dominated by LoS links, more and more recent works started to investigate the performance of UAV-aided communications with Rician fading model, which comprises a deterministic LoS component with a random multipath component, and is suitable for characterizing A2G channels with less shadowing but non-negligible small-scale fading. In [ㅁ], the number of users that could be served by the UAV was maximized in Rician fading, under the constraint of users' minimum rate-coverage probability requirements. In [ [20], the outage probability of the UAV-aided data collection with WPT was minimized in Rician fading, where the fixed UAV's altitude was assumed. In [2]], the outage probability in Rician fading was minimized, where a UAV acted as a relay to assist information transmission from BS to SNs. In [22], the coverage area of the UAV-assisted communication was maximized, with a given outage probability threshold in Rician fading. In [R2], the minimum average data collection rate from all SNs was maximized, where the angled-dependent Rician fading model was adopted. In [24], the outage probability was minimized and the coverage region was maximized in Rician fading, where the UAV's altitude was optimized.

\section{Motivation and Contributions}

However, in aforementioned works, see e.g., [एव]- [20], the Rician factor was assumed as a constant without considering the effect of elevation angle on the channel gain. As UAV's elevation angle has great impact on the A2G link quality, it has been reported that omitting the effect of the elevation angle on the Rician channel modeling causes the analyzing bias to prac-

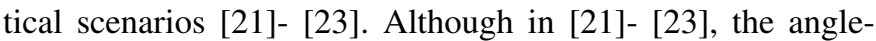
dependent Rician factor was considered, the same path loss exponent was adopted with UAV at different altitudes, which may be still too ideal, since it was pointed out in [[25] that the path loss exponent decreases as the UAV moves up, rather than being a constant. Later, in [24], both the angle-dependent Rician factor and angle-dependent path loss exponent were adopted to analyze the UAV-aided communications via $A 2 G$ channels, while however, only the information transmission was discussed and the WPT was not involved.

To fill this gap, this paper studies the outage and coverage performance of UAV-aided data collection with WPT for wireless powered network in Rician fading channels, where in order to take the effect of UAV's elevation angle into account, the angle-dependent Rician factor and angle-dependent path loss exponent are adopted. It is noticed that in [प्G]- [24], it was assumed that the SN could always perform data uploading no matter how much energy was harvested. That is, the information transmission outage was assumed to occur only in the information transmission stage. However, in practical systems, only when the harvested energy surpass the circuit activation threshold, the data transmission can be triggered at the SNs. Thus, in our work, the overall outage probability is discussed with considering the effect of the circuit activation threshold on the system information outage probability.

The main contributions of this paper are summarized as follows.

1) For the UAV-aided WPT and data collection network, the closed-form expressions of energy-constrained outage probability and rate-constrained outage probability are derived, where it is assumed that when the harvested energy at the SN cannot surpass its circuit activation threshold or the received data rate at UAV falls below a minimal rate requirement threshold, the information outage occurs. And then, a overall system information outage probability is analyzed to characterize the system outage and coverage performance.

2) In order to further enhance the system performance, an optimization problem is formulated to minimize the overall outage probability via optimizing UAV's elevation angle and time splitting (TS) factor, subject to the maximum and minimum elevation angle constraints and the TS factor constraint. Since the derived closed-form expression of the overall outage probability is non-convex, an alternating optimization (AO)based algorithm with Golden-section (GS) based linear search method is proposed to find the joint global optimal solution.

3) As the UAV has different coverage regions when it hovers at different altitudes, another optimization problem is also formulated to maximize the coverage range by optimizing UAV's altitude, while the outage probability within the coverage region being lower than a given tolerable threshold. As the coverage radius is a pseudo-concave function of elevation angle, the optimum UAV's elevation angle is derived by Karush-Kuhn-Tucker (KKT) conditions.

4) Monte Carlo simulations verify the accuracy of the derived closed-form expression of the system outage probability and the semi-closed-form expressions of the optimum UAV's elevation angle and TS factor. It is observed that there exist a 


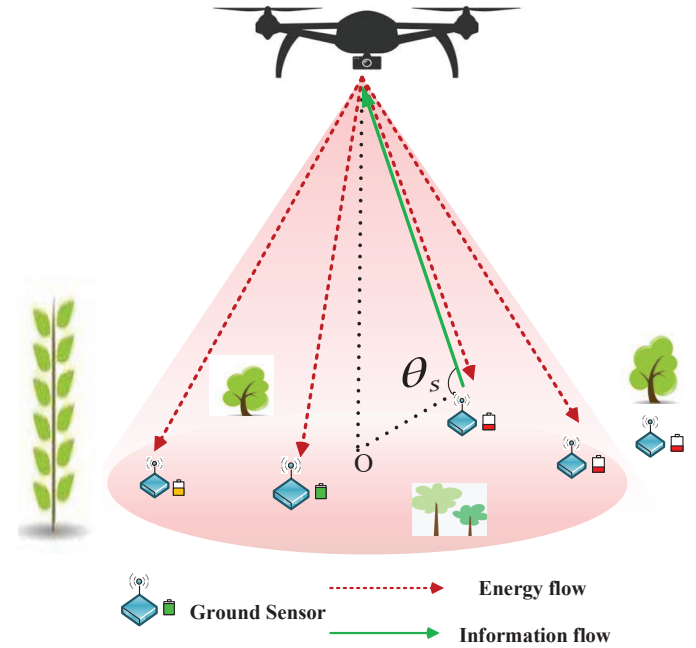

Fig. 1: UAV-aided wireless power transfer and data collection network.

unique optimum UAV's elevation angle and TS factor, which can achieve the minimum system outage probability. And it is also shown that significant performance gain can be obtained by using the joint optimization scheme. Besides, the larger the tolerable outage probability threshold is, the larger the coverage radius of UAVs communication is, and the smaller the UAV's elevation angle is.

The remainder of this paper is organized as follows. In section II, a UAV-aided wireless power transfer and data collection network is introduced. In section III, the system outage probability is analyzed. In section IV, an optimization problem of minimizing outage probability is formulated and solution method is presented. In section $\mathrm{V}$, the maximum coverage area of the UAV is derived. In section VI, simulation results are provided. In section VII, the conclusion is addressed.

\section{SYSTEM MODEL}

\section{A. Network Model}

As shown in Fig. 四, a UAV-aided wireless power transfer and data collection network is considered, where multiple SNs are deployed to monitor the environment. As the $\mathrm{SNs}$ are energy-limited, which do not have enough energy to perform computing and communication operations, a rotary-wing UAV is employed to wirelessly charge them and then schedules the SNs to upload their sensed data to the UAV, where the time-division multiple access (TDMA) protocol is employed to avoid the inter-user interference caused by data uploading among multiple SNs. That is, only one SN is scheduled to offload data within one time block, and the outage probability for the scheduled SN to transmit data to the UAV will be analyzed.

Moreover, the downlink WPT and the uplink data offloading are performed over the same frequency band. Thus, in order to avoid the interference between the WPT and data uploading, the UAV firstly charges the SNs and then schedules one SN to transmit data. Specifically, for a normalized time block with $T$ $=1 \mathrm{~s}$, the first interval of duration $(1-\rho) T$ is assigned UAV to charge the SN with $\rho \in(0,1)$ being the time division scaling factor, and the remaining interval of duration $\rho T$ is assigned the $\mathrm{SN}$ to upload data to UAV.

A polar coordinate system is employed to describe the horizontal positions of SNs and the UAV, where the plane projection of the UAV is located at the pole of the polar coordinate system, and the UAV's coordinate is denoted as $\left(0,0, h_{u}\right)$ with the UAV hovering at an altitude $h_{u}$ of several meters. The polar coordinates of the SN currently served by the UAV is denoted as $\left(g_{s}, \varphi_{s}\right)$, where $g_{s}$ represents the distance from the $\mathrm{SN}$ to the pole, and $\varphi_{s}$ denotes the polar angle of the $\mathrm{SN}$. Besides, a disk area centered at the projection of the UAV on the ground, denoted as $\mathcal{O}$, is used to describe the coverage area of the UAV. It is assumed that only when the SN located within the disk area, it can be served by the UAV.

\section{B. A2G Channel Model}

As the UAV is usually dispatched to serve ground users in the open suburban scenarios at an altitude of several meters, the LoS links are likely to be established between the UAV and the SNs. Therefore, the LoS-dominant channel model is employed in this paper, which is characterized by two kinds of channel fading, i.e., the large-scale path-loss fading and the small-scale Rician fading. In this work, both the downlink and the uplink channels are assumed to experience the large-scale path loss fading and the independent and identically distributed small-scale Rician fading, which can be modeled by

$$
\mathrm{h}_{\mathrm{us}}=\sqrt{\mathrm{L}_{\mathrm{us}}} \Omega_{\mathrm{us}},
$$

where $\mathrm{L}_{\mathrm{us}}$ is the large-scale average channel power gain, accounting for signal attenuation of the path loss, which is given by

$$
\mathrm{L}_{\mathrm{us}}=\beta d_{\mathrm{us}}^{-\alpha_{\mathrm{us}}}
$$

with $\beta$ being channel gain parameter depending on antenna characteristics and average channel attenuation, $d_{\mathrm{us}}=$ $\sqrt{\mathrm{h}_{u}^{2}+g_{s}^{2}}$ being the distance between the UAV and SN, and $\alpha_{\mathrm{us}}$ being the path loss exponent. $\Omega_{\mathrm{us}}$ is used to describe the small-scale fading, which as mentioned previously, is modeled by Rician distribution, following the weighted noncentral- $\chi^{2}$ distribution with two degrees of freedom and $\mathbb{E}\left[\left|\Omega_{\mathrm{us}}\right|^{2}\right]=1$. Thus, the probability distribution function (PDF) of $\Omega_{\mathrm{us}}$ is [26]

$$
\begin{array}{r}
f_{\Omega_{\mathrm{us}}}(\varpi)=\frac{\left(K_{\mathrm{us}}+1\right) e^{-K_{\mathrm{us}}}}{\Omega_{\mathrm{us}}} \exp \left(\frac{-\left(K_{\mathrm{us}}+1\right) \varpi}{\Omega_{\mathrm{us}}}\right) \\
I_{0}\left(2 \sqrt{\frac{K_{\mathrm{us}}\left(K_{\mathrm{us}}+1\right) \varpi}{\Omega_{\mathrm{us}}}}\right), \varpi \geq 0,
\end{array}
$$

where $K_{\text {us }}$ is the Rician factor defined as the ratio of the power in the LoS component to the power in the multipath scatters, and $I_{0}(\cdot)$ is the zero-order modified Bessel function of the first kind. Particularly, when $K_{\text {us }}=0$, (B]) is reduced to an exponential distribution indicating a Rayleigh fading channel; when $K_{\text {us }} \rightarrow \infty$, the channel converges to an additive white Gaussian noise (AWGN) channel.

Moreover, in terms of ( $(2), \alpha_{\mathrm{us}}$ can be expressed by

$$
\alpha_{\mathrm{us}}=\frac{\mathrm{L}_{\mathrm{dB}}\left(d_{\mathrm{us}}\right)+\beta_{\mathrm{dB}}}{10 \log \left(d_{\mathrm{us}}\right)},
$$

where $L_{\mathrm{dB}}$ is the path loss in $\mathrm{dB}$ and $\beta_{\mathrm{dB}}$ is the channel power gain parameter at the reference distance of one meter in 
$\mathrm{dB}$. It was reported that the path loss exponent $\alpha_{\mathrm{us}}$ decreases as the UAV moves up [25]. In order to characterize the angle-dependent path loss exponent, the A2G channel model presented in [27] is adopted, so the path loss w.r.t the elevation angle $\theta_{s}$ and $d_{\mathrm{us}}$ in $\mathrm{dB}$ is given by

$$
\mathrm{PL}_{1}\left(\theta_{s}, d_{\mathrm{uS}}\right)=\left(\eta_{\mathrm{LoS}}-\eta_{\mathrm{NLoS}}\right) \mathbb{P}_{\mathrm{LoS}}\left(\theta_{s}\right)+\operatorname{PL}_{\mathrm{NLoS}}\left(d_{\mathrm{uS}}\right),
$$

where $\eta_{\mathrm{LoS}}$ and $\eta_{\mathrm{NLoS}}$ denote the excessive path losses of the LoS propagation and the NLoS propagation from UAV to the $\mathrm{SN}$, respectively, and the LoS probability is mathematical modeled by [28] $\mathbb{P}_{\mathrm{LoS}}\left(\theta_{s}\right)=\frac{1}{1+a_{1} e^{-b_{1}\left(\theta_{s}-a_{1}\right)}}$, and $\operatorname{PL}_{\mathrm{NLOS}}\left(d_{\mathrm{us}}\right)=20 \log \left(\frac{4 \pi f_{c} d_{\mathrm{us}}}{c}\right)+\eta_{\mathrm{NLoS}}$, with $f_{c}$ being the system frequency and $c$ being the speed of light. By replacing $\mathrm{L}_{\mathrm{dB}}\left(d_{\mathrm{us}}\right)$ with $\mathrm{PL}_{1}\left(\theta_{s}, d_{\mathrm{us}}\right)$, the angle-dependent path loss exponent is further re-expressed by [24]

$$
\begin{aligned}
& \alpha_{\mathrm{us}}\left(\theta_{s}\right)=\sum_{n=1}^{N} \frac{\eta_{\mathrm{LoS}}-\eta_{\mathrm{NLoS}}}{10 N \log \left(d_{n}\right)} \mathbb{P}_{\mathrm{LoS}}\left(\theta_{s}\right)+\sum_{n=1}^{N} \frac{\mathrm{PL}_{\mathrm{NLoS}}\left(d_{\mathrm{us}}\right)+\beta_{\mathrm{dB}}}{10 N \log \left(d_{n}\right)} \\
& =a_{2} \cdot \mathbb{P}_{\mathrm{LoS}}\left(\theta_{s}\right)+b_{2},
\end{aligned}
$$

where $a_{2}=\sum_{n=1}^{N} \frac{\eta_{\mathrm{LoS}}-\eta_{\mathrm{NLoS}}}{10 N \log \left(d_{n}\right)}$ and $b_{2}=\sum_{n=1}^{N} \frac{\mathrm{PL}_{\mathrm{NLoS}}\left(d_{\mathrm{us}}\right)+\beta_{\mathrm{dB}}}{10 N \log \left(d_{n}\right)}$, which are determined by environmental characteristics (e.g., suburban, urban, dense urban) and the system frequency, and $\eta_{\mathrm{LoS}}, \eta_{\mathrm{NLoS}}$ and $\mathrm{PL}_{\mathrm{NLoS}}\left(d_{\mathrm{us}}\right)$ can be obtained by real measurement. From (ब), it can be observed the angle-dependent path loss exponent $\alpha\left(\theta_{s}\right)$ includes the NLoS parameters, which implies that our considered LoS-dominant channel model in (D) is able to reflect both the LoS and NLoS propagation effects of the A2G channel.

Moreover, according to [RQ], when the UAV communicates with the SN at different altitudes, different Rician factors should be adopted to efficiently characterize the A2G channel, and $K_{\mathrm{us}}\left(\theta_{s}\right)$ is modeled by an exponential function of $\theta_{s}$, i.e.,

$$
K_{\mathrm{us}}\left(\theta_{s}\right)=a_{3} \cdot e^{b_{3} \theta_{s}},
$$

where the unit of $\theta_{s}$ in $(\mathbb{D})$ is in radian, $a_{3}$ and $b_{3}$ are environment and frequency dependent constant parameters with $a_{3}=k_{0}$ and $b_{3}=\frac{2}{\pi} \ln \left(\frac{k_{\frac{\pi}{2}}}{k_{0}}\right) . k_{0}=K_{\text {us }}(0)$ and $k_{\frac{\pi}{2}}=K_{\mathrm{us}}\left(\frac{\pi}{2}\right)$ are determined by measurements in a concrete scenario [29]. It is a fact that a larger $\theta_{s}$ corresponds to a strong LoS link while a smaller $\theta_{s}$ represents a severer multipath conditions.

\section{Energy Harvesting and Data Collection}

In the downlink WPT phase with duration of $(1-\rho) T$, the harvested energy at the $\mathrm{SN}$ is given by

$$
E_{\mathrm{S}}=\eta P_{u}\left|\mathrm{~h}_{\mathrm{us}}\right|^{2}(1-\rho) T
$$

where $\eta$ is the energy conversion efficiency of converting the received RF signals into direct current (DC) signals for energy harvesting, and $P_{u}$ is UAV's transmit power.

In the uplink phase with duration of $\rho T$, the $\mathrm{SN}$ uploads data to the UAV, the available transmit power of the $\mathrm{SN}$ to upload data is

$$
P_{\mathrm{s}}=\eta P_{u}\left|\mathrm{~h}_{\mathrm{us}}\right|^{2}\left(\frac{1-\rho}{\rho}\right)
$$

Consequently, the instantaneous received SNR at the UAV is given by

$$
\gamma_{\mathrm{su}}=\frac{\eta P_{u}(1-\rho)}{N_{0} \rho}\left|\mathrm{h}_{\mathrm{us}}\right|^{2}\left|\mathrm{~h}_{\mathrm{su}}\right|^{2},
$$

where $N_{0}$ is the noise power, and $\mathrm{h}_{\mathrm{su}}$ is the channel coefficient from the SN to the UAV.

\section{Outage Probability Analysis}

For the considered UAV-aided wireless power transfer and data collection network, the data uploading outage may occur in both the energy harvesting phase and the data collection phase. In the energy harvesting phase, when the harvested energy is not enough to surpass the circuit activation threshold, the uplink data uploading will not be started. In this case, the information transmission outage may occur. In the data uploading phase, when the received data rate at UAV falls below a threshold $C_{\mathrm{th}}$, the information outage will also occur. Thus, the overall system outage probability is given by

$$
P_{\text {out }}=P_{\text {out }}^{(\mathrm{EH})}+\left(1-P_{\text {out }}^{(\mathrm{EH})}\right) P_{\text {out }}^{(\text {Info })},
$$

where $P_{\text {out }}^{(\mathrm{EH})}$ is used to describe the energy outage probability, which is given by

$$
\begin{aligned}
& P_{\mathrm{out}}^{(\mathrm{EH})}=\mathbb{P}\left(E_{\mathrm{S}} \leq E_{\mathrm{th}}\right) \\
& =\mathbb{P}\left(\left|\Omega_{\mathrm{us}}\right|^{2} \leq \frac{E_{\mathrm{th}} d_{\mathrm{us}} \alpha_{\mathrm{us}}}{\eta P_{u} \beta(1-\rho) T}\right) \\
& =1-Q_{1}\left(\mathrm{x}, \mathrm{y}_{\mathrm{eh}}\right),
\end{aligned}
$$

with

$$
\left\{\begin{array}{l}
\mathrm{x}=\sqrt{2 K_{\mathrm{us}}} \\
\mathrm{y}_{\mathrm{eh}}=\sqrt{\frac{2\left(K_{\mathrm{us}}+1\right) E_{\mathrm{th}} d_{\mathrm{us}}{ }^{\alpha} \mathrm{us}}{\eta P_{u} \beta(1-\rho) T}},
\end{array}\right.
$$

and $Q_{1}(\cdot)$ is the first order Marcum $Q$-function [26]. $P_{\text {out }}^{(\text {Info })}$ is used to describe the information outage probability, which is given by

$$
\begin{aligned}
& P_{\text {out }}^{(\text {Info }}=\mathbb{P}\left(\rho \log _{2}\left(1+\gamma_{\mathrm{su}}\right) \leq C_{\mathrm{th}}\right) \\
& =\mathbb{P}\left(\left.\frac{\beta^{2} \eta P_{u}(1-\rho)}{N_{0} d_{\mathrm{us}}{ }^{2 \alpha_{\mathrm{us}} \rho} \mid \Omega_{\mathrm{us}}}\right|^{2} \times\left|\Omega_{\mathrm{su}}\right|^{2} \leq 2^{\frac{C_{\mathrm{th}}}{\rho}}-1\right) \\
& =\int_{0}^{\infty} \mathbb{P}\left[\sqrt{\frac{\eta P_{u}(1-\rho)}{N_{0} \rho}} \frac{\beta}{d_{\mathrm{us}} \alpha_{\mathrm{us}}}\left|\Omega_{\mathrm{us}}\right|^{2} \leq \frac{2^{\frac{C \mathrm{th}}{\rho}}-1}{y}\right] \times \\
& \frac{\partial}{\partial y}\left(\mathbb{P}\left[\sqrt{\frac{\eta P_{u}(1-\rho)}{N_{0} \rho}} \frac{\beta}{d_{\mathrm{us}}{ }^{\alpha_{\mathrm{us}}}}\left|\Omega_{\mathrm{su}}\right|^{2} \leq y\right]\right) d y \\
& =1-\int_{0}^{\infty} Q_{1}\left(\sqrt{2 K_{\mathrm{us}}}, \sqrt{\frac{2\left(K_{\mathrm{us}}+1\right)\left(2^{\frac{C_{\mathrm{th}}}{\rho}}-1\right) d_{\mathrm{us}}{ }^{\alpha_{\mathrm{us}}}}{y \beta} \sqrt{\frac{N_{0} \rho}{\eta P_{u}(1-\rho)}}}\right) \\
& \times \frac{\partial}{\partial y}\left[1-Q_{1}\left(\sqrt{2 K_{\mathrm{us}}}, \sqrt{\frac{2\left(K_{\mathrm{us}}+1\right) y d_{\mathrm{us}}{ }^{\alpha_{\mathrm{us}}}}{\beta} \sqrt{\frac{N_{0} \rho}{\eta P_{u}(1-\rho)}}}\right)\right] d y .
\end{aligned}
$$

In order to provide some deep analytical insights on the system outage performance, a tight exponential-type approximation [B]] is adopted to fit $Q_{1}(\cdot)$, which is expressed by

$$
Q_{1}(a, b) \approx \exp \left(-e^{\phi(a)} b^{\varphi(a)}\right),
$$

where $\phi(a)$ and $\varphi(a)$ have different expressions with different values of $a$ [Bत] and [B]]. For example, when $10 \leq a \leq 8000$, the polynomial expressions of $\phi(a)$ and $\varphi(a)$ can be given by [B]]

$$
\phi(a) \triangleq-3.0888 \times 10^{-10} a^{6}+1.8362 \times 10^{-7} a^{5}
$$




$$
\begin{aligned}
& -3.7185 \times 10^{-5} a^{4}+3.4103 \times 10^{-3} a^{3} \\
& -0.1624 a^{2}-1.4318 a+0.7409 \\
\varphi(a) \triangleq & 5.1546 \times 10^{-11} a^{6}-3.1961 \times 10^{-8} a^{5} \\
+ & 6.3859 \times 10^{-6} a^{4}-5.4159 \times 10^{-4} a^{3} \\
+ & 1.9833 \times 10^{-2} a^{2}+0.9044 a+0.9439
\end{aligned}
$$

Lemma 1: With the tight exponential-type approximation for Marcum $Q$-function in ([5), the overall system outage probability $P_{\text {out }}$ is given by

$$
\begin{aligned}
& P_{\text {out }}= \\
& 1+2 Q_{1}\left(\mathrm{x}, \mathrm{y}_{\mathrm{eh}}\right) \ln \left(Q_{1}\left(\mathrm{x}, \mathrm{y}_{\text {info }}\right)\right) \mathbf{K}_{\mathbf{1}}\left(-2 \ln \left(Q_{1}\left(\mathrm{x}, \mathrm{y}_{\text {info }}\right)\right)\right)
\end{aligned}
$$

with

$$
\mathrm{y}_{\text {info }}=\sqrt{\frac{2\left(K_{\mathrm{us}}+1\right) d_{\mathrm{us}}{ }^{{ }_{\mathrm{us}}}}{\beta} \sqrt{\frac{N_{0} \rho\left(2^{\frac{C_{\mathrm{th}}}{\rho}}-1\right)}{\eta P_{u}(1-\rho)}}},
$$

and $\mathbf{K}_{\mathbf{1}}(\cdot)$ being the first order modified Bessel function of second kind.

Proof: The proof can be found in Appendix $\Theta$.

\section{PRoblem FORMULATION AND SOLUTION METHODS}

As the UAV can adjust its altitude to establish a better communication link to the SN, the effect of elevation angle on the outage probability is analyzed, which may provide some useful insights on how to efficiently deploy UAVs. Moreover, a smaller TS factor $\rho$ indicates that a longer time is allocated to the $\mathrm{SNs}$ for $\mathrm{EH}$ and the less time is remained for data uploading, while a larger TS factor $\rho$ indicates that the SNs are assigned more time to upload data but may not harvest enough energy due to the less remaining time, which implies that there is a trade-off between $\mathrm{EH}$ and data uploading. In order to achieve the best trade-off, the TS factor $\rho$ is required to be optimized to yield the minimum overall system outage probability. To this end, an optimization problem is formulated to minimize the system outage probability via optimizing UAV's elevation angle $\theta_{s}$ and TS factor $\rho$, which is mathematically given by

$$
\begin{array}{ll}
\mathbf{P}_{\mathbf{A}}: \min _{\left\{\theta_{\mathbf{s}}, \rho\right\}} P_{\text {out }} \\
\text { s.t. } & \mathrm{C}_{1}: \theta_{\min } \leq \theta_{s} \leq \theta_{\max }, \\
& \mathrm{C}_{2}: 0 \leq \rho \leq 1,
\end{array}
$$

where $\mathrm{C}_{1}$ indicates the maximum and the minimum elevation angle constraints, and $\mathrm{C}_{2}$ means the TS factor is constrained within the range of $(0,1)$.

Due to the presence of highly non-linear terms and the coupling variables of $\theta_{s}$ and $\rho$ in $P_{\text {out }}$, problem $\mathbf{P}_{\mathbf{A}}$ is nonconvex, which is difficult to tackle. Therefore, an AO-based algorithm with GS-based linear search method is proposed, which is able to find a good optimal solution. Specifically, $\mathbf{P}_{\mathbf{A}}$ is divided into two subproblems to optimize $\theta_{s}$ and $\rho$ separately and iteratively, where GS-based method is used to find the optimal solution of each subproblem.

\section{A. Optimizing $\theta_{s}$ with given $\rho$}

With a given $\rho$, the outage probability $P_{\text {out }}$ is non-convex of $\theta_{s}$. However, it is found that $P_{\text {out }}$ is pseudoconvex in $\theta_{s}$ in the feasible region defined by $\mathrm{C}_{1}$, which is addressed in the following Lemma.

Lemma 2: The outage probability $P_{\text {out }}$ is a pseudo-convex function of UAV's elevation angle $\theta_{s}$.

Proof: The proof can be found in Appendix B].

As a result, the analytical expression of the optimal $\theta_{s}$ for minimizing $P_{\text {out }}$ can be obtained.

Lemma 3: The optimal $\theta_{s}^{*}$ of problem $\mathbf{P}_{\mathbf{A}}$ for minimizing the outage probability is given by

$$
\begin{gathered}
\left(\theta_{s}^{*}, \nu_{1}^{*}, \nu_{2}^{*}\right)= \\
\begin{cases}\left(\theta_{s, c}, 0,0\right), & \theta_{\min } \leq \theta_{s, c} \leq \theta_{\max }, \\
\left(\theta_{\max }, \nu_{1, B}, 0\right), & \theta_{\min } \leq \theta_{\max } \leq \theta_{s, c}, \\
\left(\theta_{\min }, 0, \nu_{2, B}\right), & \theta_{s, c} \leq \theta_{\min } \leq \theta_{\max },\end{cases}
\end{gathered}
$$

where the critical point $\theta_{s, c}$ can be calculated in terms of

$$
\begin{aligned}
& \sqrt{\sqrt{\frac{N_{0} \rho\left(2^{\frac{C_{\mathrm{th}}}{\rho}}-1\right)}{\eta P_{u}(1-\rho) \beta^{2}}}\left[\frac{g_{s}}{\cos \left(\theta_{s, c}\right)}\right]^{\alpha_{\mathrm{us}}\left(\theta_{s, c}\right)}}\left[\frac{K^{\prime}\left(\theta_{s, c}\right)}{K\left(\theta_{s, c}\right)+1}+\right. \\
& \left.\alpha_{\mathrm{us}}^{\prime} \ln \left(\frac{g_{s}}{\cos \left(\theta_{s, c}\right)}\right)+\alpha_{\mathrm{us}}\left(\theta_{s, c}\right) \tan \left(\theta_{s, c}\right)\right]=\frac{\mathcal{A}+2}{\mathcal{A}^{2}+2} \frac{K^{\prime}\left(\theta_{s, c}\right)}{K\left(\theta_{s, c}\right)+1},
\end{aligned}
$$

with $\mathcal{A}=\left(\frac{E_{\mathrm{th}}{ }^{2}}{\eta P_{u}(1-\rho) N 0 \rho\left(2^{\frac{C_{\mathrm{th}}}{\rho}}-1\right)}\right)^{\frac{1}{4}}$, and the Lagrange multipliers $\nu_{1, B}$ and $\nu_{2, B}$ are respectively given by

$$
\begin{aligned}
& \nu_{1, B}=\left[\frac{2 Q_{1}\left(\mathrm{x}_{\max }, \mathcal{A} \mathrm{y}_{\max }\right) \mathrm{z}_{\max } \mathbf{K}_{\mathbf{0}}\left(\mathrm{z}_{\max }\right)}{Q_{1}\left(\mathrm{x}_{\max }, \mathrm{y}_{\max }\right)} \frac{\partial Q_{1}\left(\mathrm{x}_{\max }, \mathrm{y}_{\max }\right)}{\partial \theta_{s}}\right. \\
& \left.+\frac{\partial Q_{1}\left(\mathrm{x}_{\max }, \mathcal{A}_{\left.\mathrm{y}_{\max }\right)}\right)}{\partial \theta_{s}} \mathrm{z}_{\max } \mathbf{K}_{\mathbf{1}}\left(\mathrm{z}_{\max }\right)\right] .
\end{aligned}
$$

and

$$
\begin{aligned}
& \nu_{2, B}=-\left[\frac{2 Q_{1}\left(\mathrm{x}_{\min }, \mathcal{A} \mathrm{y}_{\min }\right) \mathrm{x}_{\min } \mathbf{K}_{\mathbf{0}}\left(\mathrm{z}_{\min }\right)}{Q_{1}\left(\mathrm{x}_{\min }, \mathrm{y}_{\min }\right)} \frac{\partial Q_{1}\left(\mathrm{x}_{\min }, \mathrm{y}_{\min }\right)}{\partial \theta_{s}}\right. \\
& \left.+\frac{\partial Q_{1}\left(\mathrm{x}_{\min }, \mathcal{A}_{\min }\right)}{\partial \theta_{s}} \mathrm{z}_{\min } \mathbf{K}_{\mathbf{1}}\left(\mathrm{z}_{\min }\right)\right] .
\end{aligned}
$$

Proof: The proof can be found in Appendix $\mathrm{C}$

It is observed that, due to the presence of highly non-linear terms in (एD) , it is not possible to obtain the explicit analytic solution for $\theta_{s, c}$. Thus, the GS-based linear search technique [34] is adopted to find the critical point $\theta_{s, c}$ by numerically solving (एप). For clarity, the presented GS-based method is summarized in Algorithm $\mathbb{W}$, where the function $f_{\theta}$ used in the method is defined as

$$
\begin{aligned}
& f_{\theta}=\sqrt{\sqrt{\frac{N_{0} \rho\left(2^{\frac{C_{\mathrm{th}}}{\rho}}-1\right)}{\eta P_{u}(1-\rho) \beta^{2}}}\left[\frac{g_{s}}{\cos \left(\theta_{s}\right)}\right]^{\alpha_{\mathrm{us}}}}\left[\frac{K^{\prime}\left(\theta_{s}\right)}{K\left(\theta_{s}\right)+1}+\right. \\
& \left.\alpha_{\mathrm{us}}^{\prime} \ln \left(\frac{g_{s}}{\cos \left(\theta_{s}\right)}\right)+\alpha_{\mathrm{us}} \tan \left(\theta_{s}\right)\right]-\frac{\mathcal{A}+2}{\mathcal{A}^{2}+2} \frac{K^{\prime}\left(\theta_{s}\right)}{K\left(\theta_{s}\right)+1} .
\end{aligned}
$$

\section{B. Optimizing $\rho$ with given $\theta_{s}$}

It is observed that constraint $\mathrm{C}_{2}$ is affine, which defines a convex set $\mathcal{S}$ w.r.t $\rho$, and $\mathrm{C}_{2}$ is kept implicit because it will be never satisfied at strict equality. Therefore, the minimal outage probability $P_{\text {out }}$ in $\mathbf{P}_{\mathbf{A}}$ over $\mathcal{S}$ can be obtained by letting $\frac{\partial P_{\text {out }}}{\partial \rho}=0$ due to the pseudo-convexity of $P_{\text {out }}$ in $\rho$, which is stated in Lemma 因. 


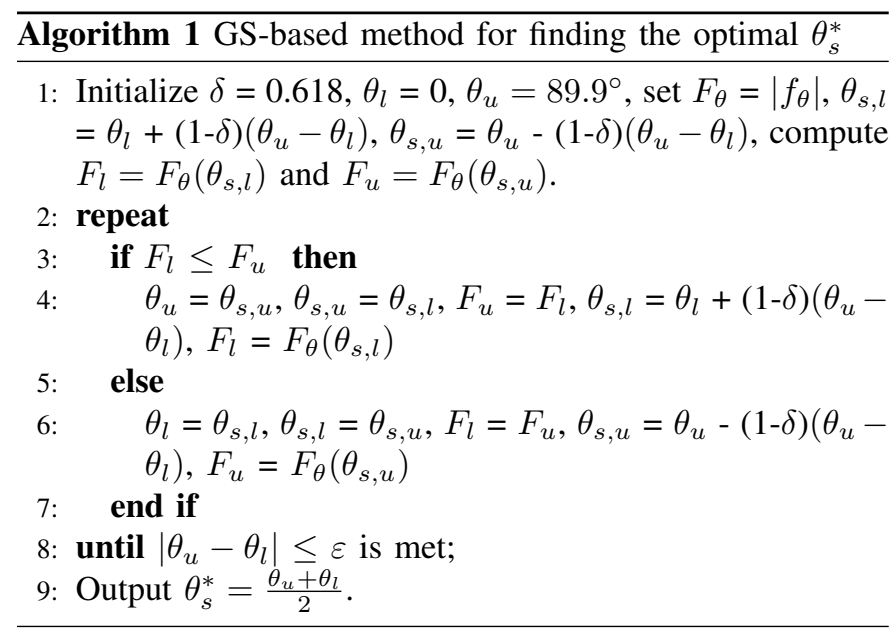

Lemma 4: The outage probability $P_{\text {out }}$ in $\mathbf{P}_{\mathbf{A}}$ is pseudoconvex in $\rho$, and the optimal $\rho^{*}$ is approximately given by

$$
\left(\frac{E_{\mathrm{th}}}{\eta P_{u}(1-\rho)}\right)^{\mathcal{B}}=\mathcal{G}_{2}^{\frac{\mathcal{B}}{2}-1} \frac{N_{0}}{\eta P_{u}}\left(\frac{2^{\frac{C_{\mathrm{th}}}{\rho}} C_{\mathrm{th}} \ln 2(1-\rho)-\rho\left(2^{\frac{C_{\mathrm{th}}}{\rho}}-1\right)}{\rho(1-\rho)}\right) \text {. }
$$

Proof: The proof can be found in Appendix DD. Similar to (एप), there is no explicit analytic expression for $\rho^{*}$. Thus, the GS-based method in Algorithm $\mathbb{W}$ also can be adopted to find the optimal $\rho^{*}$ by numerically solving ([23).

For a special case, when the energy threshold is extremely small, the impact of energy on the system outage probability can be neglected. As a result, the optimal TS factor $\rho_{\text {info }}^{*}$ to problem $\mathbf{P}_{\mathbf{A}}$ is presented by the following Lemma.

Lemma 5: With $E_{\mathrm{th}}=\frac{\eta P_{u} \beta(1-\rho)\left[Q_{1}^{-1}\left(\sqrt{2 K_{\mathrm{us}}}, 1\right)\right]}{2\left(K_{\mathrm{us}}+1\right) d_{\mathrm{us}}{ }^{\alpha_{\mathrm{us}}}}$, the optimal $\rho_{\text {info }}^{*}$ is given by

$$
\rho_{\text {info }}^{*}=\frac{C_{\mathrm{th}} \ln 2}{1+C_{\mathrm{th}} \ln 2+\mathcal{W}\left(-e^{-C_{\mathrm{th}} \ln 2-1}\right)},
$$

where $\mathcal{W}(\chi)$ is the Lambert $\mathcal{W}$ function with $\mathcal{W}(\chi) e^{\mathcal{W}(\chi)}=$ $\chi$.

Proof: The proof can be found in Appendix $\mathbb{D}$.

\section{Joint optimization of $\theta_{s}$ and $\rho$}

It is noticed that $P_{\text {out }}$ is pseudoconvex in $\theta_{s}$ with fixed $\rho$, which is proved in Appendix $\mathbb{B}$, and $P_{\text {out }}$ is pseudoconvex in $\rho$ with fixed $\theta_{s}$, which is proved in Appendix $\mathbb{D}$. Thus, according to the concept of bi-pseudoconvexity shown in the definition $\mathbf{\square}$ [38], $P_{\text {out }}$ in $\mathbf{P}_{\mathbf{A}}$ is bi-pseudoconvex of $\theta_{s}$ and $\rho$ over a bi-convex set defined by the constraints $\mathrm{C}_{1}$ and $C_{2}$.

Definition 1: A function $f(x, y)$ with $x \in X$ and $y \in Y$, defined over a bi-convex set $B \subset X \times Y$, is called a bipseudoconvex if upon fixing $x=\bar{x}, f_{x}(y)=f(\bar{x}, y)$ is pseudoconvex over $Y$, and fixing $y=\bar{y}, f_{y}(x)=f(x, \bar{y})$ is pseudoconvex over $X$.

Proposition 1: The presented Algorithm 2 is able to find the global optimal solution to problem $\mathbf{P}_{\mathbf{A}}$.

Proof: Since the outage probability function is bipseudoconvex w.r.t the elevation angle and TS factor over a bi-convex set as analyzed previously, according to [BQ], the proposed AO-based algorithm is able to find the optimal solution.

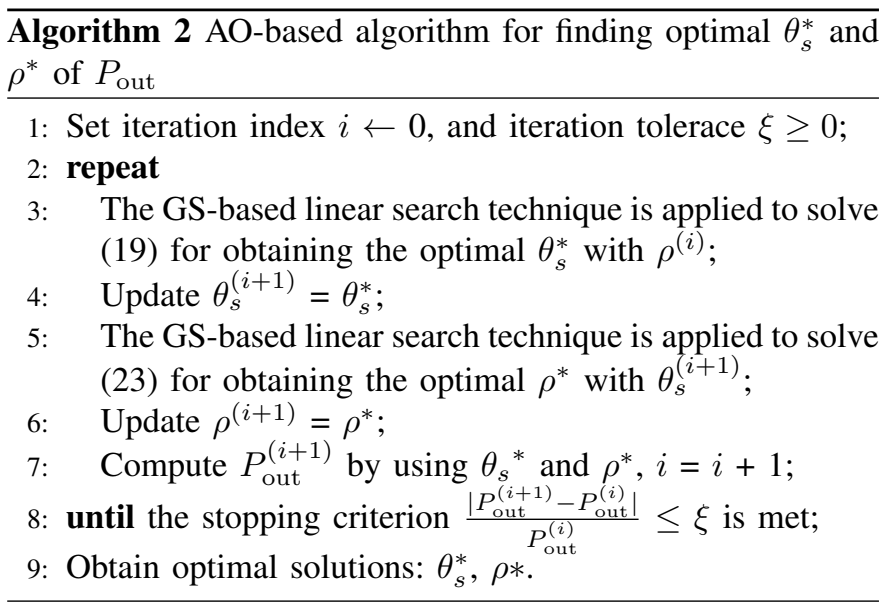

\section{Complexity analysis of the proposed algorithm}

The proposed Algorithm 2 runs in an iterative manner. Let $N_{\text {c }}$ be the iteration number for finding the optimal solution, and in each iteration, the GS-based method is executed to find the optimal elevation angle $\theta_{s}^{*}$ and the optimal TS factor $\rho^{*}$ in step 3 and step 5, respectively. For the GS-based method, only a sum, a comparison and a rounding are executed, and the feasible region is narrowed by ratio $(1-\delta)$, where $\delta$ denotes the golden section ratio. As such, the iteration number of the GS-based method is about $\left\lceil\frac{\log \left(\frac{\varepsilon[i]}{s_{u}[i]-s_{l}[i]}\right)}{\log (\delta)}\right\rceil$, with $i=1$ indicating that the optimal $\theta_{s}$ is found in step 3 , and $i=2$ indicating that the optimal $\rho$ is found in step 5 , where $\varepsilon[i]$ denotes the maximum tolerance, $\left[s_{l}[i], s_{u}[i]\right]$ denotes the initial search interval with $s_{l}[i]$ being the lower bound and $s_{u}[i]$ being the upper bound, and $\lceil\cdot\rceil$ returns the smallest integer greater than or equal to its numeric argument. As a result, the overall computational complexity of the proposed solution approach is approximately on the order of $O\left(N_{\mathrm{c}}\left(\left\lceil\frac{\log \left(\frac{\varepsilon[1]}{s_{u}[1]-s_{l}[1]}\right)}{\log (\delta)}\right\rceil+\left\lceil\frac{\log \left(\frac{\varepsilon[2]}{s_{u}[2]-s_{l}[2]}\right)}{\log (\delta)}\right\rceil\right)\right)$

\section{Maximum Coverage Area}

In the multi-SN scenarios, multiple $\mathrm{SNs}$ desire to be well covered and served by the UAV. Since different altitudes of the hovering UAV yields different coverage results and efficiency, the optimal altitude of UAV is expected to be found to maximize the coverage under the constraint of the predefined tolerable outage probability of the SNs. That is, if $P_{\text {out }}\left(h_{u}, R_{\mathrm{us}}\right) \leq P_{\mathrm{th}}$, the reliable A2G link can be established between the UAV and the SN, which is within the circle area with the radius of $R_{\mathrm{us}}$, where $P_{\mathrm{th}}$ denotes the tolerable outage probability threshold; Otherwise, the information transmission outage between the SN and the UAV may occur. Thereby, for a given altitude $h_{u}$, the boundary of the UAV's coverage area implies that

$$
P_{\text {out }}\left(h_{u}, R_{\text {us }}\right)=P_{\text {th }},
$$

where $R_{\mathrm{us}}$ is the coverage radius. In order to maximize the coverage range, an optimization problem is formulated, which is given by

$$
\mathbf{P}_{\mathbf{B}}: \max _{\left\{\theta_{\mathbf{s}}\right\}} R_{\mathrm{us}}
$$




$$
\text { s.t. } \mathrm{C}_{1}: \theta_{\min } \leq \theta_{s} \leq \theta_{\max } .
$$

Since

$$
\left\{\begin{array}{l}
h_{u}=d_{\mathrm{us}} \sin \left(\theta_{s}\right), \\
R_{\mathrm{us}}=d_{\mathrm{us}} \cos \left(\theta_{s}\right),
\end{array}\right.
$$

with $d_{\mathrm{us}}=\left[\frac{\beta \mathrm{y}_{\text {info }}{ }^{2}}{\left(\mathrm{x}^{2}+2\right) \sqrt{\mathcal{G}_{2}}}\right]^{\frac{1}{\alpha_{\mathrm{us}}}}$ and $\mathcal{G}_{2}=\frac{N_{0} \rho\left(2^{\frac{C_{\mathrm{th}}}{\rho}}-1\right)}{\eta P_{u}(1-\rho)}$, solving problem $\mathbf{P}_{\mathbf{B}}$ lies in finding the analytical expression between $\mathrm{y}_{\text {info }}$ and $x$, which is stated in Lemma [6.

Lemma 6: For a given $\mathrm{x}=\sqrt{2 K_{\mathrm{us}}}$, the relationship between $\mathrm{y}_{\text {info }}$ and $\mathrm{x}$ is given by

$$
\mathrm{y}_{\text {info }}=\left\{\begin{array}{l}
\mathrm{y}_{0} e^{\frac{\left(\frac{\mathcal{A}+2}{\mathcal{A}^{2}+2}\right) \mathrm{x}^{2}}{4}}, \mathrm{x} \leq \mathrm{x}_{\mathrm{c}} \\
\left(\frac{\mathcal{A}+2}{\mathcal{A}^{2}+2}\right) \mathrm{x}-\frac{3 \ln \left(\frac{\mathrm{x}}{(\mathcal{A}+2) \mathrm{x}+\left(\mathcal{A}^{2}+2\right) c}\right)}{2\left(\mathcal{A}^{2}+2\right) c}+c \\
\mathrm{x} \geq \mathrm{x}_{\mathrm{C}} \wedge c \neq 0 \\
\left(\frac{\mathcal{A}+2}{\mathcal{A}^{2}+2}\right) \mathrm{x}+\frac{3}{2(\mathcal{A}+2) \mathrm{x}}, x \geq x_{c} \wedge c=0
\end{array}\right.
$$

where $x_{c}$ is the intersection of the sub-functions.

Proof: The proof can be found in Appendix $\mathbb{E}$.

Lemma 7: The coverage radius $\ln \left(R_{\mathrm{us}}\right)$ in $\mathbf{P}_{\mathbf{B}}$ is pseudoconcave in $\theta_{s}$, and the optimal $\theta_{s}^{*}$ is given by

$$
\begin{gathered}
\left(\theta_{s}^{*}, \lambda_{1}^{*}, \lambda_{2}^{*}\right)= \\
\left\{\begin{array}{cl}
\left(\theta_{s}^{\operatorname{cov}}, 0,0\right), & \theta_{\min } \leq \theta_{s}^{\operatorname{cov}} \leq \theta_{\max } \\
\left(\theta_{\max }, \lambda_{1}, 0\right), & \theta_{\min } \leq \theta_{\max } \leq \theta_{s}^{\operatorname{cov}} \\
\left(\theta_{\min }, 0, \lambda_{2}\right), & \theta_{s}^{\operatorname{cov}} \leq \theta_{\min } \leq \theta_{\max }
\end{array}\right.
\end{gathered}
$$

where the critical point $\theta_{s}^{\text {cov }}$ can be calculated based on the following equation

$$
\begin{aligned}
& 2 \mathrm{x}^{\prime}\left(\frac{\mathcal{A}+2}{(\mathcal{A}+2) \mathrm{x}+\left(\mathcal{A}^{2}+2\right) c}-\frac{1}{\mathrm{x}}\right)= \\
& \left.\alpha_{\mathrm{us}}^{\prime} \ln \left(d_{\mathrm{us}}\left(\theta_{s}^{\mathrm{cov}}\right)\right)+\alpha_{\mathrm{us}}\left(\theta_{s}^{\mathrm{cov}}\right)\right) \tan \left(\theta_{s}^{\mathrm{cov}}\right),
\end{aligned}
$$

with $\mathrm{x}^{\prime}$ and $\alpha_{\mathrm{us}}^{\prime}$ indicating the derivative functions w.r.t $\theta_{s}$.

Proof: The proof can be found in Appendix $\mathbb{E}$.

\section{Simulation Results}

In this section, some simulation results are provided to evaluate the obtained analytical results and the presented optimization schemes. The impact of network parameters on the overall system outage probability are also discussed via simulations. The simulation settings are similar to [20], [24] and [B]], and the detailed parameter settings are summarized in Table I.

Fig. $\square$ shows that the analytical results of the overall system outage probability obtained in terms of Lemma $\square$ are in a good conformity with the simulation results obtained by Monte Carlo method for different UAV's altitudes. Moreover, it is observed that there exists an optimal UAV's altitude $h_{u}^{*}$ that minimizes the outage probability. When $h_{u}$ increases within the range of $\left[h_{u}, h_{u}^{*}\right]$, the gain brought by decreasing path loss exponent $\alpha_{\mathrm{us}}$ and the increasing Rician factor $K_{\mathrm{us}}$ is more significant than the loss caused by the increased link length, so the overall system outage probability is reduced. However, when $h_{u}$ exceeds $h_{u}^{*}$, the loss caused by the increased link length may be larger than the gain caused by the reduced path
TABLE I: Simulation Parameters

\begin{tabular}{l|c|c}
\hline \hline Parameters & Notation & Values \\
\hline \hline The transmit power of UAV & $P_{u}$ & $30 \mathrm{dBm}$ \\
The channel power gain & $\beta_{0}$ & $-20 \mathrm{~dB}$ \\
The system noise power & $N_{0}$ & $-110 \mathrm{dBm}$ \\
Rician factors related parameters & $k_{0}$ & $5 \mathrm{~dB}$ \\
Rician factors related parameters & $k_{\frac{\pi}{2}}$ & $15 \mathrm{~dB}$ \\
Path loss exponent related parameters & $a_{2}$ & -1.5 \\
Path loss exponent related parameters & $b_{2}$ & 3.5 \\
UAV-ground channel parameters & $a_{1}$ & $0.136 \mathrm{~dB}$ \\
UAV-ground channel parameters & $b_{1}$ & $11.95 \mathrm{~dB}$ \\
Energy harvesting efficiency & $\eta$ & 0.8 \\
Time period & $T$ & $1 \mathrm{~s}$ \\
Data rate threshold & $C_{\mathrm{th}}$ & $1 \mathrm{bit} / \mathrm{s} / \mathrm{Hz}$ \\
Energy activation threshold limit & $E_{\mathrm{th}}$ & $10^{-6} \mathrm{Watt}$ \\
\hline
\end{tabular}

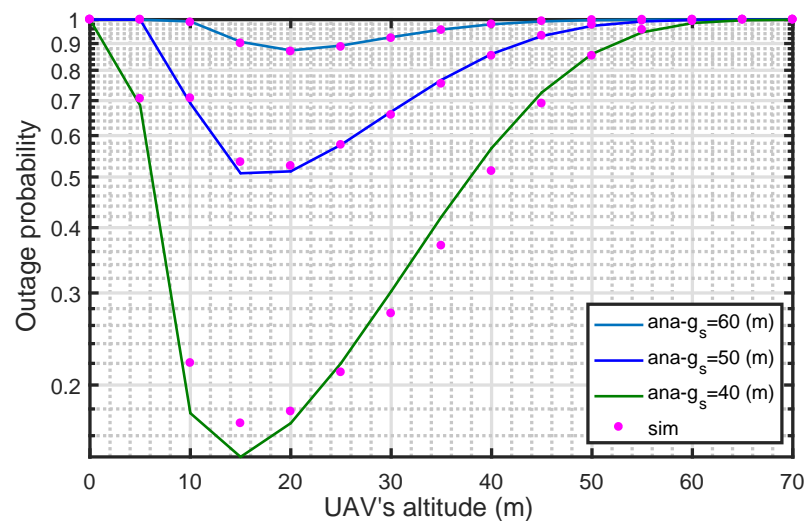

Fig. 2: Outage probability versus UAV's altitude.

loss $\alpha_{\mathrm{us}}$ and the increased Rice factor $K_{\mathrm{us}}$ with increasing $h_{u}$, which results in deteriorating the overall system outage probability. Besides, with UAV located at the same altitude, the farther the SN's location is, the larger the overall system information outage probability is.

Fig. B shows the analytical results of the overall system information outage probability versus the system TS factor. Moreover, it can be seen that as $\rho$ increases, the overall information outage probability initially decreases and then increases, showing the existence of an optimum $\rho^{*}$ for achieving the minimum overall system outage probability. Besides, the smaller the energy threshold $E_{\mathrm{th}}$ is, the smaller the overall outage probability is, and the less time allocated for energy harvesting, which leads to a larger $\rho$ for data uploading.

Fig. 团 depicts the overall system outage probability versus the UAV's altitude and TS factor $\rho$ in a 3-D pattern. It can be observed that the overall system outage probability is pseudoconvex w.r.t UAV's altitude and TS factor, respectively, which is consistent with Lemma $\square$ and Lemma $\mathbb{\text { G }}$ and there is a unique optimum UAV's altitude $h_{u}^{*}$ and an optimal TS factor $\rho^{*}$ to minimize the outage probability, which is marked by the red point in Fig. 团.

Fig. 5(a) depicts the analytical solution of $\theta_{s}^{*}$ obtained by Lemma $\square$, which closely matches the exact value obtained by the Monte Carlo method. It is shown that the larger the SN's location $g_{s}$ is, the smaller the UAV's elevation angle is. The reason may be that when $g_{s}$ is relatively small, a relatively large UAV's elevation angle results in the reduction of $\alpha_{\mathrm{us}}$ and 


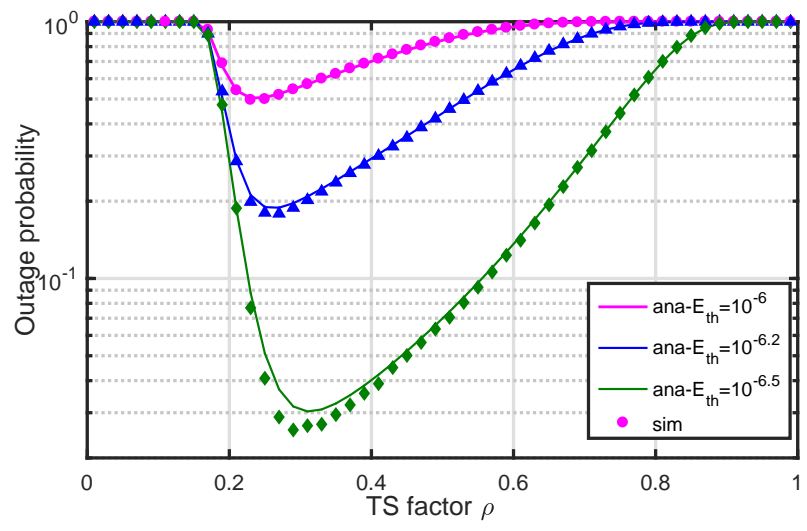

Fig. 3: Outage probability versus TS factor $\rho$.

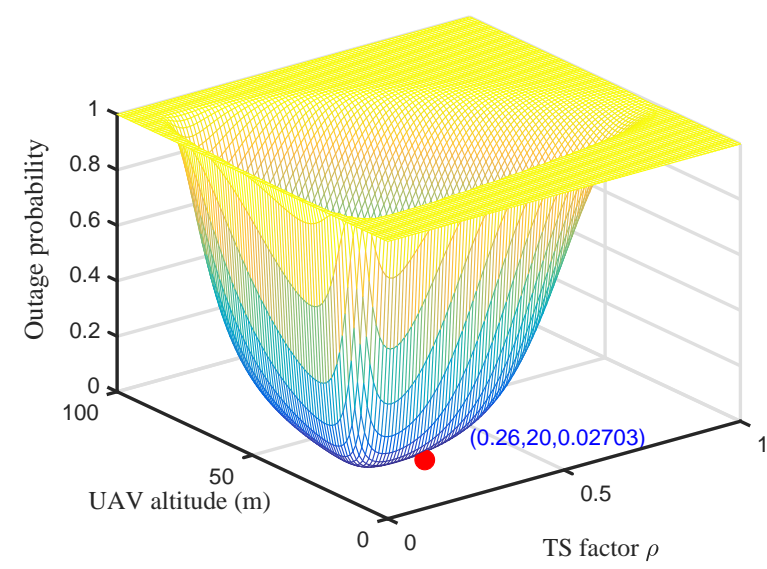

Fig. 4: Outage probability versus UAV's altitude and TS factor.

the increment of $K_{\mathrm{us}}$, which bring more performance gain to the communication over A2G channels. When $g_{s}$ is relatively large, the communication link length has a dominant impact on the overall system outage probability. In this case, the performance gain brought by the reduced path loss exponent $\alpha_{\text {us }}$ and the increased Rician factor $K_{\text {us }}$ becomes less noticeable than the performance loss caused by increased link length. Fig. 5(b) depicts the optimal $\rho^{*}$ obtained by Lemma $⿴$ t versus $E_{\text {th }}$, which closely matches the exact value obtained by Monte Carlo method. It shows that when the energy threshold $E_{\text {th }}$ is relatively small, the TS factor $\rho^{*}$ is approximately equal to the optimal solution obtained by Lemma [. Moreover, with the increment of $E_{\mathrm{th}}$, the TS factor $\rho$ decreases, since in this case the SN requires more time to harvest energy to trigger its data uploading.

Fig. 1 plots the outage probability obtained by the proposed Algorithm 2 with different initial parameter settings. It can be seen that Algorithm 2 has a good convergence behavior and it is able to converge to the global optimal solution obtained by the exhaustive search with different initial parameter settings, which is consistent with Proposition 1.

Fig. $\square$ plots the overall system outage probability versus the energy threshold $E_{\mathrm{th}}$ with four schemes, where the scheme with fixed $\rho$ and $\theta_{s}$ is denoted as Benchmark I, and the scheme with $\rho$ optimized and UAV's elevation angle fixed is denoted

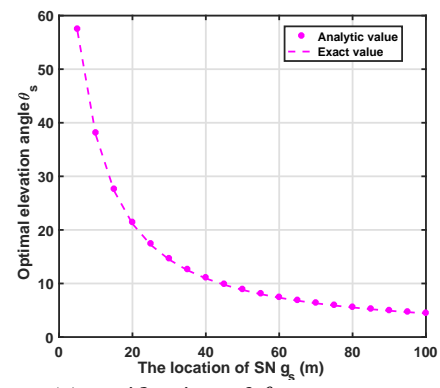

(a) verification of $\theta_{s}$ versus $g_{s}$

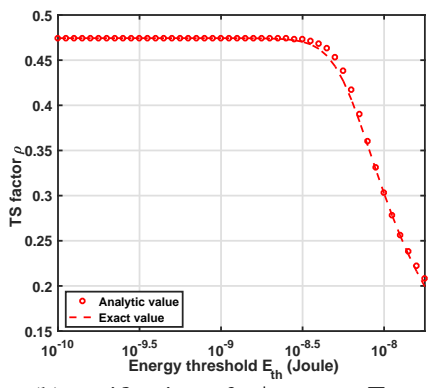

(b) verification of $\rho^{*}$ versus $E_{\mathrm{th}}$
Fig. 5: The analytical solution of $\theta_{s}^{*}$ and $\rho^{*}$ versus the Monte Carlo simulation.

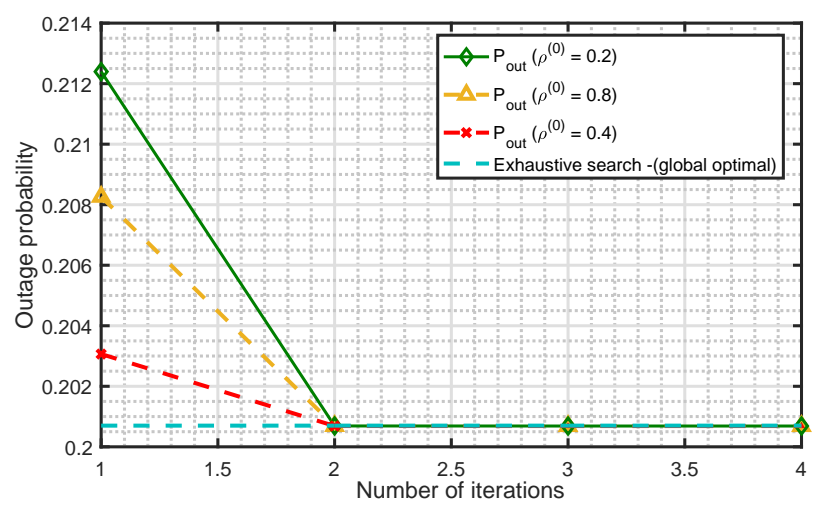

Fig. 6: Outage probability versus different initial parameters of TS factor.

as Benchmark II, and the scheme with $\theta_{s}$ optimized and $\rho$ fixed is denoted as Benchmark III, and the scheme with $\rho$ and $\theta_{s}$ jointly optimized is denoted as AO-(our proposed), which is achieved by the AO-based algorithm. The performance gap between $\mathrm{AO}$ and Benchmark I demonstrates the gain brought by joint optimization of TS factor and UAV's elevation angle, and the performance gap between $\mathrm{AO}$ and Benchmark II demonstrates the gain brought by optimizing UAV's elevation angle, and the performance gap between AO and Benchmark III demonstrates the gain brought by optimizing TS factor. It shows that the joint optimization scheme fully exploits the advantages of optimizing $\rho$ and $\theta_{s}$, which enables more efficient RF-EH and WIT, achieving the minimal overall system outage probability. Moreover, the performance gain gap firstly increases and then decreases with the increment of energy threshold, since there is no more gain can be obtained when the energy threshold is relatively large.

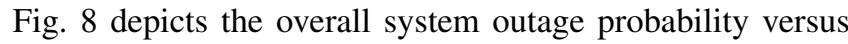
the UAV's transmit power $P_{u}$ obtained by the aforementioned four schemes. It can be observed that as $P_{u}$ increases, the overall system outage probability decreases, where however the declining rate gradually becomes slow. Moreover, our presented AO scheme always achieve the lowest overall system outage probability than other three benchmark ones.

Fig. 9 plots the overall system outage probability versus the rate threshold $C_{\mathrm{th}}$ obtained by the four different schemes. It is observed that as $C_{\text {th }}$ increases, the overall system outage probability increases, where $\mathrm{AO}$ scheme achieves the lowest 


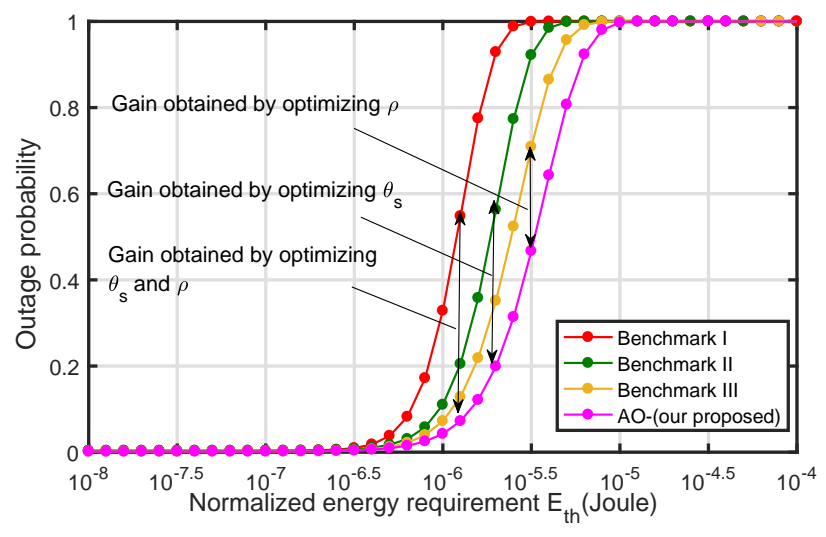

Fig. 7: Outage probability versus energy threshold $E_{\mathrm{th}}$.

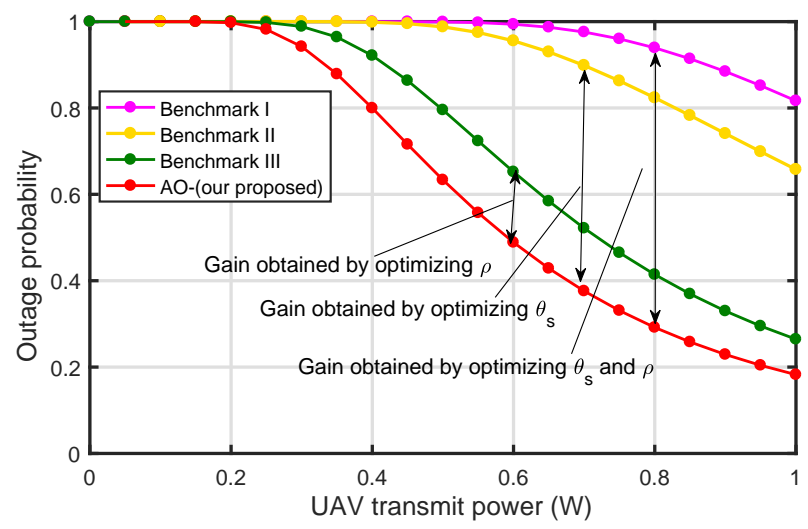

Fig. 8: Outage probability versus UAV's transmit power $P_{u}$.

outage probability than other three benchmark ones. Moreover, when the $C_{\mathrm{th}}$ is relatively small, the system performance gain obtained by optimizing $\rho$ is relatively large, which results in the overall system outage probability of Benchmark II being better than that of Benchmark III, while however, when $C_{\mathrm{th}}$ is relatively large, the optimization of $\theta_{s}$ has a more significant impact on the system performance.

Fig. [1 plots the outage probability versus the UAV's altitude, where the results obtained by our proposed design and two benchmarks, i.e., Benchmark I and Benchmark II are compared. Benchmark I represents the design of the angledependent path loss exponent with fixed Rician factor, and Benchmark II represents the design of the angle-dependent Rician factor with fixed path loss exponent. It is observed that the outage probability of the three designs first decreases and then increases with the increment of the UAV altitude. Moreover, our proposed design achieves the minimal outage probability, because in our proposed design, the angle-dependent Rician factor and the angle-dependent path loss exponent are both taken into account, which leads to a higher UAV altitude for fully exploiting the gains brought by decreasing path loss exponent and increasing Rician factor in terms of reducing the outage probability.

Fig. $\left[\right.$ depicts the optimal $\theta_{s}$ and $\rho$ versus rate threshold $C_{\text {th }}$, energy threshold $E_{\text {th }}$ and UAV's transmit power $P_{u}$. It shows that the optimal $\theta_{s}$ decreases with the increment of $C_{\mathrm{th}}$ and $E_{\mathrm{th}}$, because a lower $\theta_{s}$ leads to a shorter

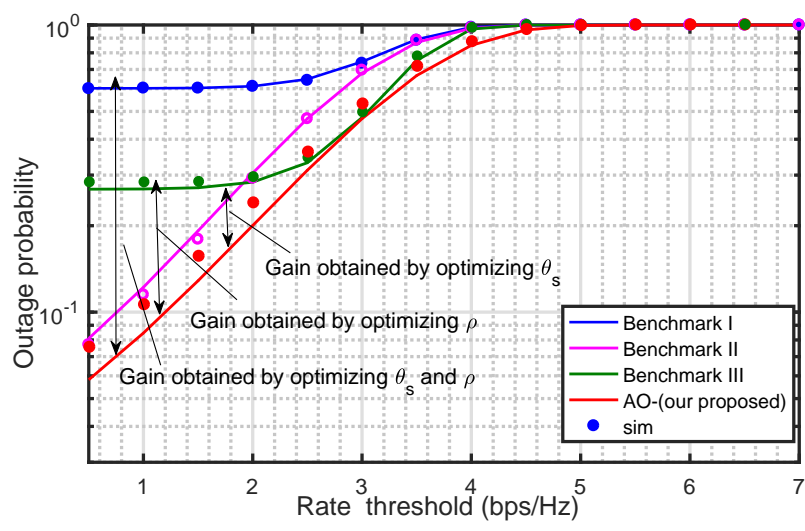

Fig. 9: Outage probability versus rate threshold $C_{\mathrm{th}}$.

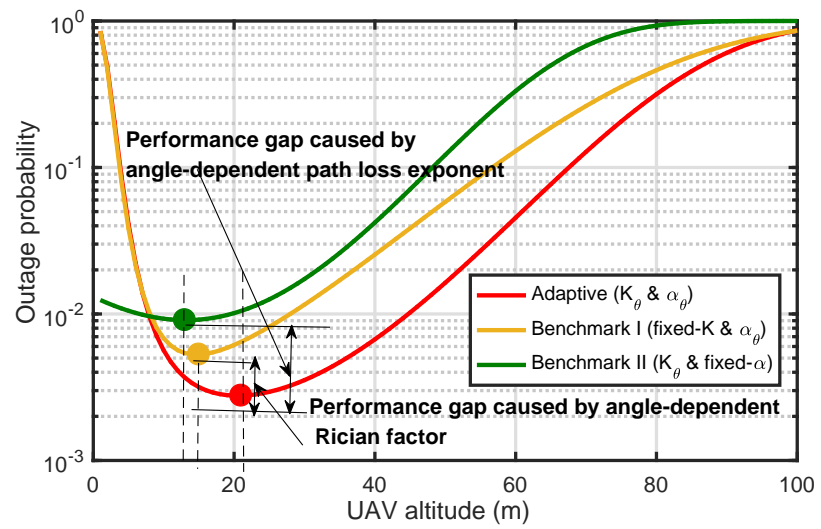

Fig. 10: The outage probability versus the UAV's altitude with different designs.

communication link length with given SN's location, such that the outage probability can be minimized. Moreover, the optimal $\theta_{s}$ increases with the increment of transmit power $P_{u}$, because when the system resource is enough, it tends to strive more benefit of increased Rician factor and decreased pass loss exponent bringing by a larger $\theta$ for minimizing the outage probability. That is, a larger $C_{\mathrm{th}}$ and $E_{\mathrm{th}}$ or a lower $P_{u}$ corresponds to a smaller $\theta_{s}$ to minimize the outage probability, which indicates that the effect of communication link length on the outage probability is more significant than that of Rician factor and path loss exponent. Additionally, as $C_{\text {th }}$ and $P_{u}$ increase, the optimal TS factor $\rho$ increases, but as $E_{\text {th }}$ increase, the optimal $\rho$ decreases.

Fig. 12 shows that there exists two different $\theta_{s}$ to achieve the same coverage radius before arriving at the optimum UAV's elevation angle to maximize the coverage area. Because a smaller UAV's elevation angle with less gain of increased path loss exponent and reduced Rice factor may lead to a shorter communication link. On the contrary, a larger UAV's elevation angle with more gain of reduced path loss exponent and increased Rice factor may lead to a longer communication link. Therefore, there is an optimal equilibrium point to find the optimal elevation angle to maximize the coverage area. Besides, when the UAV's elevation angle and TS factor are optimized, the system coverage performance can be maximized as marked by the red circle in Fig. 12. 

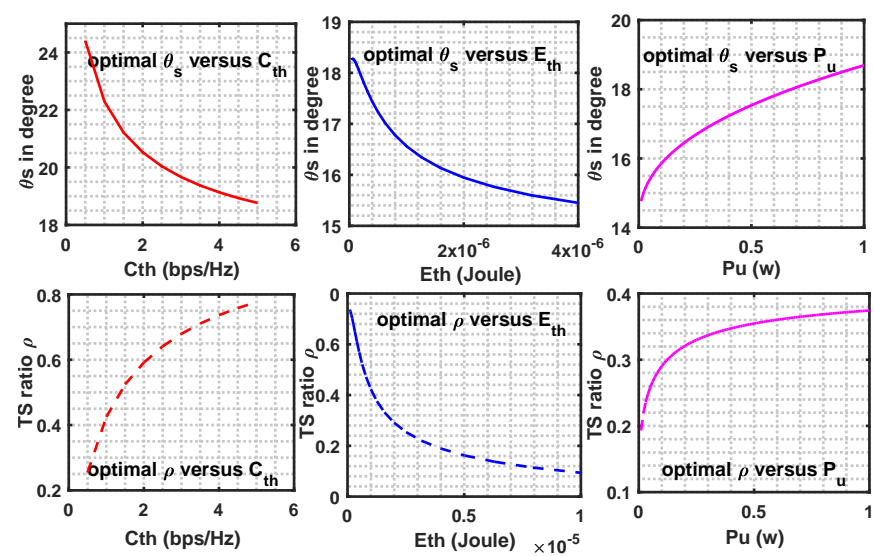

Fig. 11: Optimal $\theta_{s}$ and $\rho$ to minimize the outage probability.

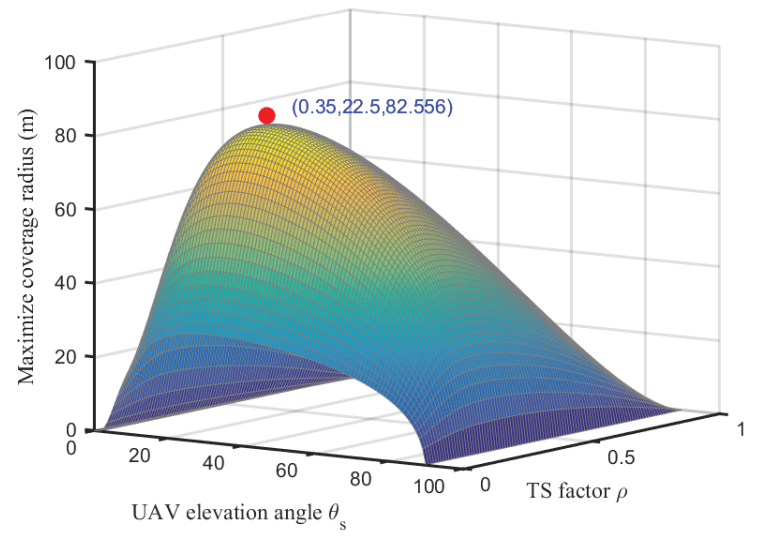

Fig. 12: Coverage radius versus UAV's altitude and TS factor.

Fig. [3] depicts the maximum coverage area of the UAV with different tolerable outage probability threshold $P_{\mathrm{th}}$. It can be observed that a smaller tolerable outage probability threshold $P_{\text {th }}$ leads to a more stringent reliability link constraint, which reduces the coverage radius of the UAVs communication. Moreover, the larger the tolerable outage probability threshold $P_{\text {th }}$ is, the larger the coverage radius is, and the smaller the UAV's elevation angle is. Because the impact of the communication link length on the outage probability becomes greater with the increment of coverage radius, and a smaller UAV's elevation angle can slightly compensate for the severe losses caused by the increased link length and yield a larger coverage radius.

Fig. 14(a) depicts the UAV's maximum coverage radius versus rate threshold $C_{\mathrm{th}}$. It shows that the larger the $C_{\mathrm{th}}$ is, the smaller the achievable coverage radius is, which implies that the scope of high-quality communication services provided by the UAV is limited. It is noticed that in Fig.14(b), as the $C_{\mathrm{th}}$ increases, the optimal elevation angle $\theta_{s}$ for maximizing the coverage radius decreases. The reason may be that when the communication quality requirement is stringent, to ensure that the outage probability is lower than a tolerable threshold, a smaller elevation angle is necessary to reduce the link length and maximize the coverage radius, which is consistent with Fig.미. Moreover, a larger coverage radius can be obtained with a smaller $C_{\mathrm{th}}$, which corresponds to a larger $\theta_{s}$. As

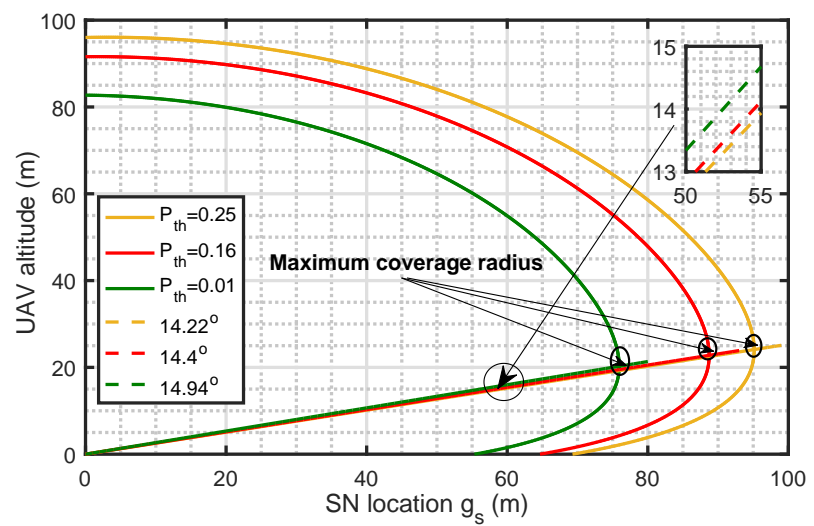

Fig. 13: Maximum coverage area with different $P_{\text {th }}$.

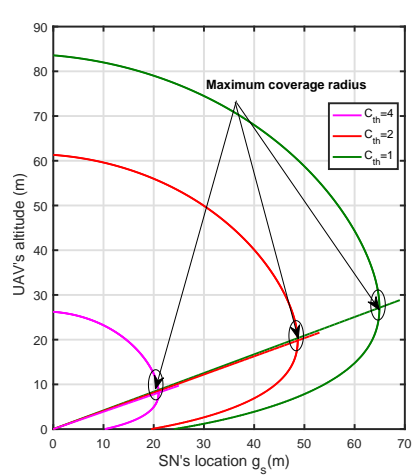

(a)

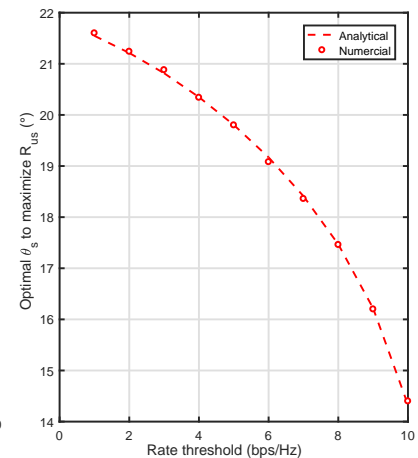

(b)
Fig. 14: Maximum coverage radius versus $C_{\text {th }}$ and optimal $\theta_{s}$.

the performance gain brought by increased Rician factor and decreased path loss exponent with a larger $\theta_{s}$ can be relatively fully exploited and compensate for the loss caused by the increased link length, which is able to ensure the outage probability lower than the tolerable threshold.

\section{CONCLUSION}

This paper studied the TDMA-aware UAV-aided wireless power transfer and data collection network, where one SN was scheduled to be served by the UAV in one transmission block. An optimization problem was formulated to minimize the overall outage probability between the scheduled SN and the UAV by optimizing UAV's elevation angle and the TS factor. Since the problem is non-convex and has no known solution, an AO-based algorithm with GS-based linear search method was proposed to find the global optimal solution. Besides, in order to explore the maximum coverage area of the UAV for a given tolerable outage probability, another optimization problem was also formulated to maximize the coverage range by optimizing UAV's elevation angle. By using KKT conditions, the closed-form solution of the optimal elevation angle was derived. Monte Carlo simulations verify the accuracy of the derived closed-form expression of the overall outage probability and the semi-closed-form expressions of the optimum UAV's elevation angle and TS factor. It indicates that there exist a unique optimum UAV's elevation angle and the TS factor to achieve the minimum system outage probability, and significant performance gain can be obtained by using 
our proposed optimization scheme. The developed theoretical results can be useful to the design of UAV-aided wireless communication systems with WPT. Besides, the trajectory design of the UAV across multiple time slots with the outage probability and coverage probability constraints is really an interesting and open issue, which will be investigated in future.

\section{APPENDIX A}

\section{The Proof of Lemma $\square$}

Firstly, by letting $\mathrm{x}=\sqrt{2 K_{\mathrm{us}}}, \quad G=$ $\frac{2\left(K_{\mathrm{us}}+1\right) d_{\mathrm{us}}{ }^{{ }_{\mathrm{us}}}}{\beta} \sqrt{\frac{N_{\mathrm{o}} \rho}{\eta P_{u}(1-\rho)}}$ and $\mathcal{B}=\frac{\varphi(x)}{2}$, and using the approximation in (표) one has

$$
\begin{aligned}
& P_{\text {out }}^{\text {(Info) }}=1-\int_{0}^{\infty} \exp \left(-e^{\phi(x)}\left(\frac{G\left(2 \frac{C_{\text {th }}}{\rho}-1\right)}{y}\right)^{\mathcal{B}}\right) \times \\
& \frac{\partial}{\partial y}\left[1-\exp \left(-e^{\phi(x)}(G y)^{\mathcal{B}}\right)\right] d y \\
& =1-\int_{0}^{\infty} \exp \left(-e^{\phi(x)}\left(\frac{G\left(2^{\frac{C_{\text {th }}}{\rho}}-1\right)}{y}\right)^{\mathcal{B}}\right) \times \\
& \exp \left(-e^{\phi(x)}(G y)^{\mathcal{B}}\right)\left(e^{\phi(x)} \mathcal{B} G^{\mathcal{B}} y^{\mathcal{B}-1}\right) d y \\
& =1-e^{\phi(x)} G^{\mathcal{B}} \int_{0}^{\infty} \exp \left(-e^{\phi(x)} G^{\mathcal{B}}\left(\frac{\left(2^{\frac{C_{\text {th }}}{\rho}}-1\right)^{\mathcal{B}}}{y^{\mathcal{B}}}+y^{\mathcal{B}}\right)\right) d y^{\mathcal{B}} \\
& =1-2 e^{\phi(x)} G^{\mathcal{B}}\left(2^{\frac{C_{\text {th }}}{\rho}}-1\right)^{\frac{\mathcal{B}}{2}} \mathbf{K}_{\mathbf{1}}\left(2 e^{\phi(x)} G^{\mathcal{B}}\left(2^{\frac{C_{\text {th }}}{\rho}}-1\right)^{\frac{\mathcal{B}}{2}}\right) \\
& =1-2 e^{\phi(x)} \mathrm{y}_{\text {info }}{ }^{\varphi(x)} \mathbf{K}_{\mathbf{1}}\left(2 e^{\phi(x)} \mathrm{y}_{\text {info }} \varphi(x)\right. \\
&
\end{aligned}
$$

where $y_{\text {info }}=\sqrt{\frac{2\left(K_{\text {us }}+1\right) d_{\text {us }}{ }^{\alpha_{\text {us }}}}{\beta} \sqrt{\frac{N_{0} \rho\left(2 \frac{C_{\text {th }}}{\rho}-1\right)}{\eta P_{u}(1-\rho)}}}$. With the approximation in (디) reused, one has $-\ln \left(Q_{1}(x, y)\right) \approx$ $e^{\phi(x)} y^{\varphi(x)}$ and $P_{\text {out }}^{(\text {Info) }}$ is reexpressed by

$$
P_{\text {out }}^{\text {(Info) }}=1+2 \ln \left(Q_{1}\left(\mathrm{x}, \mathrm{y}_{\text {info }}\right)\right) \mathbf{K}_{\mathbf{1}}\left(-2 \ln \left(Q_{1}\left(\mathrm{x}, \mathrm{y}_{\text {info }}\right)\right)\right) \text {. }
$$

And then, the system outage probability $P_{\text {out }}$ is expressed by

$$
\begin{aligned}
& P_{\text {out }}=P_{\text {out }}^{(\mathrm{EH})}+\left(1-P_{\text {out }}^{(\mathrm{EH})}\right) P_{\text {out }}^{(\mathrm{Inf})} \\
& =1+2 Q_{1}\left(\mathrm{x}, \mathrm{y}_{\mathrm{eh}}\right) \ln \left(Q_{1}\left(\mathrm{x}, \mathrm{y}_{\text {info }}\right)\right) \mathbf{K}_{\mathbf{1}}\left(-2 \ln \left(Q_{1}\left(\mathrm{x}, \mathrm{y}_{\text {info }}\right)\right)\right) .
\end{aligned}
$$

Thus, the proof is completed.

\section{APPENDIX B \\ The Proof of Lemma}

Here the pseudoconvexity of $P_{\text {out }}$ in $\theta_{s}$ is proved. Firstly, the definition of pseudoconvex function is stated as follows.

A differentiable function $f: \mathbb{R}^{n} \rightarrow \mathbb{R}$, defined on a convex set $S$, is called pseudoconvex if $\forall x, y \in S$ with $x \neq y$ and $\nabla f(x)^{T}(y-x) \geq 0 \Rightarrow f(y) \geq f(x)$ [B2]. And, a pseudoconvex function $f$ has a similar property as in convex functions, which states that, if $\exists$ a critical point, i.e., $\nabla f(\bar{x})$ $=0$, then $\bar{x}$ is a global minimum point. As unimodality of a single variable function is equivalent to its pseudoconvexity, and then if a function is unimodal in $\theta_{s}$ over the convex set, it is also a pseudoconvex function of $\theta_{s}$ [B3] $]$.

Next we aim to find the critical point of $P_{\text {out }}$ by solving $\frac{\partial P_{\text {out }}}{\partial \theta_{s}}=0$. By introducing a intermediate variable $\mathcal{A}$ to represses the relationship between $\mathrm{y}_{\mathrm{eh}}$ and $\mathrm{y}_{\text {info }}$, we have

$$
\mathrm{y}_{\mathrm{eh}}=\left(\frac{E_{\mathrm{th}}{ }^{2}}{\eta P_{u}(1-\rho) N_{0} \rho\left(2^{\frac{C_{\mathrm{th}}}{\rho}}-1\right)}\right)^{\frac{1}{4}} \mathrm{y}_{\text {info }}=\mathcal{A} \mathrm{y}_{\text {info }} .
$$

With a little abuse of notations, by defining $u=Q_{1}$ (x, yinfo $_{\text {) }}$ and $z=-2 \ln (u)$ and $P_{\text {out }}$ is reexpressed by

$$
P_{\text {out }}=1-Q_{1}\left(\mathrm{x}, \mathcal{A} y_{\text {info }}\right) z \mathbf{K}_{\mathbf{1}}(z) .
$$

Following multivariable chain rule differentiation, the negative first derivative of $P_{\text {out }}$ is given by

$$
\begin{aligned}
& -\frac{\partial P_{\text {out }}}{\partial \theta_{s}}= \\
& Q_{1}\left(\mathrm{x}, \mathcal{A} \mathbf{y}_{\text {info }}\right) \frac{\partial\left[z \mathbf{K}_{1}(z)\right]}{\partial z} \frac{\partial[z]}{\partial u} \frac{\partial[u]}{\partial \theta_{s}}+\frac{\partial Q_{1}\left(\mathrm{x}, \mathcal{A} \mathrm{y}_{\text {info }}\right)}{\partial \theta_{s}} z \mathbf{K}_{\mathbf{1}}(z) .
\end{aligned}
$$

Following [B]], one has

$$
\left\{\begin{array}{l}
\mathbf{K}_{v-1}(z)=-\frac{v}{z} \mathbf{K}_{v}(z)-\mathbf{K}_{v}^{\prime}(z), \\
\frac{\partial\left[z \mathbf{K}_{1}(z)\right]}{\partial z}=\mathbf{K}_{\mathbf{1}}(z)+z \mathbf{K}_{\mathbf{1}}{ }^{\prime}(z)=-z \mathbf{K}_{\mathbf{0}}(z) .
\end{array}\right.
$$

Thus, by letting $\frac{\partial P_{\text {out }}}{\partial \theta_{s}}=0$, with $\lim _{z \rightarrow \infty} \frac{\mathbf{K}_{1}(z)}{\mathbf{K}_{\mathbf{0}}(z)}=1$ we have

$$
\begin{aligned}
& \frac{2 Q_{1}\left(\mathrm{x}, \mathcal{A} \mathrm{y}_{\text {info }}\right) z \mathbf{K}_{\mathbf{0}}(z)}{Q_{1}\left(\mathrm{x}, \mathrm{y}_{\text {info }}\right)} \frac{\partial Q_{1}\left(\mathrm{x}, \mathrm{y}_{\text {info }}\right)}{\partial \theta_{s}}+\frac{\partial Q_{1}\left(\mathrm{x}, \mathcal{A} \mathrm{y}_{\text {info }}\right)}{\partial \theta_{s}} z \mathbf{K}_{\mathbf{1}}(z)=0, \\
& -2 Q_{1}\left(\mathrm{x}, \mathcal{A}_{\left.\mathrm{y}_{\text {info }}\right)} \frac{\partial Q_{1}\left(\mathrm{x}, \mathrm{y}_{\text {info }}\right)}{\partial \theta_{s}}=Q_{1}\left(\mathrm{x}, \mathrm{y}_{\text {info }}\right) \frac{\partial Q_{1}\left(\mathrm{x}, \mathcal{A} \mathrm{y}_{\text {info }}\right)}{\partial \theta_{s}}\right.
\end{aligned}
$$

From [24] and [B]], one sees that

$$
\left\{\begin{array}{l}
Q_{1}(x, y)=e^{-\frac{x^{2}+y^{2}}{2}} I_{0}(x y), \\
\frac{\partial Q_{1}(x, y)}{\partial \theta_{s}}=\frac{\partial Q_{1}(x, y)}{\partial x} \frac{\partial x}{\partial \theta_{s}}+\frac{\partial Q_{1}(x, y)}{\partial y} \frac{\partial y}{\partial \theta_{s}}, \\
\frac{\partial Q_{1}(x, y)}{\partial x}=y e^{-\frac{x^{2}+y^{2}}{2}} I_{1}(x y), \\
\frac{\partial Q_{1}(x, y)}{\partial y}=-y e^{-\frac{x^{2}+y^{2}}{2}} I_{0}(x y) .
\end{array}\right.
$$

By substituting (B2a), (B2D), (B2C) and (B2d) into (Bل]), one has

$$
\begin{aligned}
& -2 I_{0}\left(\mathrm{x}_{\mathcal{A}} \mathrm{y}_{\text {info }}\right)\left(I_{1}\left(\mathrm{xy}_{\text {info }}\right) \frac{\partial \mathrm{x}}{\partial \theta_{s}}-I_{0}\left(\mathrm{xy}_{\text {info }}\right) \frac{\partial \mathrm{y}_{\text {info }}}{\partial \theta_{s}}\right) \\
& =I_{0}\left(\mathrm{xy}_{\text {info }}\right)\left(\mathcal{A} I_{1}\left(\mathrm{x} \mathcal{A} \mathrm{y}_{\text {info }}\right) \frac{\partial \mathrm{x}}{\partial \theta_{s}}-\mathcal{A}^{2} I_{0}\left(\mathrm{x}_{\mathcal{A}} \mathrm{y}_{\text {info }}\right) \frac{\partial \mathrm{y}_{\text {info }}}{\partial \theta_{s}}\right) \\
& \Rightarrow\left(\mathcal{A}^{2}+2\right) I_{0}\left(\mathrm{x} \mathcal{A} y_{\text {info }}\right) I_{0}\left(\mathrm{xy}_{\text {info }}\right) \frac{\partial \mathrm{y}_{\text {info }}}{\partial \theta_{s}}= \\
& \left(\mathcal{A} I_{1}\left(\mathrm{x} \mathcal{A} \mathrm{y}_{\text {info }}\right) I_{0}\left(\mathrm{xy}_{\text {info }}\right)+2 I_{1}\left(\mathrm{xy}_{\text {info }}\right) I_{0}\left(\mathrm{x} \mathcal{A} \mathrm{y}_{\text {info }}\right)\right) \frac{\partial \mathrm{x}}{\partial \theta_{s}} \\
& \Rightarrow \frac{\partial \mathrm{y}_{\text {info }}}{\partial \theta_{s}}=\left(\frac{\mathcal{A}}{\mathcal{A}^{2}+2} \frac{I_{1}\left(\mathrm{x} \mathrm{y}_{\mathrm{y}_{\text {info }}}\right)}{I_{0}\left(\mathrm{x}^{\mathrm{A}} \mathrm{y}_{\text {info }}\right)}+\frac{2}{\mathcal{A}^{2}+2} \frac{I_{1}\left(\mathrm{xy}_{\text {info }}\right)}{I_{0}\left(\mathrm{x}_{\text {info }}\right)}\right) \frac{\partial \mathrm{x}}{\partial \theta_{s}},
\end{aligned}
$$

With $\mathrm{x}=\sqrt{2 K\left(\theta_{s}\right)}$, one has $\frac{\partial \mathrm{x}}{\partial \theta_{s}}=\frac{K^{\prime}\left(\theta_{s}\right)}{\mathrm{x}}$, where $K^{\prime}(\cdot)$ indicates the derivative function of $K(\cdot)$. Moreover, according to the definition of $y_{\text {info }}$ in ([1]) we have

$$
\begin{aligned}
& 2 \ln \left(\mathrm{y}_{\text {info }}\right)= \\
& \ln \left(K_{\text {us }}+1\right)+\alpha_{\text {us }} \ln \left(\frac{g_{s}}{\cos \left(\theta_{s}\right)}\right)+\ln \left(2 \sqrt{\frac{N_{0} \rho\left(\frac{C_{\text {th }}}{\rho}-1\right)}{\eta P_{u}(1-\rho) \beta^{2}}}\right),
\end{aligned}
$$

where $d_{\mathrm{us}}=\frac{g_{s}}{\cos \left(\theta_{s}\right)}$. The derivative of $\mathrm{y}_{\text {info }}$ w.r.t $\theta_{s}$ yields

$$
\frac{\partial \text { yinfo }_{\text {ino }}}{\partial \theta_{s}}=\frac{\mathrm{y}_{\text {info }}}{2}\left[\frac{K^{\prime}\left(\theta_{s}\right)}{K\left(\theta_{s}\right)+1}+\alpha_{\mathrm{us}}^{\prime} \ln \left(\frac{g_{s}}{\cos \left(\theta_{s}\right)}\right)+\alpha_{\mathrm{us}} \tan \left(\theta_{s}\right)\right] .
$$


Assuming that $\mathrm{xy}_{\text {info }}$ is large enough, the following approximation is obtained [B]],

$$
\frac{I_{1}(x y)}{I_{0}(x y)}=1-\frac{1}{2 x y}-\frac{1}{8(x y)^{2}}+O\left[(x y)^{-3}\right] \cong 1 .
$$

And then, we have

$$
\begin{aligned}
\frac{\partial \mathrm{y}_{\text {info }}}{\partial \theta_{s}} & =\left(\frac{\mathcal{A}}{\mathcal{A}^{2}+2}\left(1-\frac{1}{2 \mathrm{x} \cdot \mathcal{A} \mathrm{y}_{\text {info }}}\right)+\frac{2}{\mathcal{A}^{2}+2}\left(1-\frac{1}{2 \mathrm{xy}_{\text {info }}}\right)\right) \frac{K^{\prime}\left(\theta_{s}\right)}{x} \\
& \approx \frac{\mathcal{A}+2}{\mathcal{A}^{2}+2} \frac{K^{\prime}\left(\theta_{s}\right)}{\mathrm{x}} .
\end{aligned}
$$

Finally, by bringing (B5]) and (B7) into (B3a), the critical point of $P_{\text {out }}$ in $\theta_{s}$ can be found in the following implicit equation

$$
\begin{aligned}
& \sqrt{\sqrt{\frac{N_{0} \rho\left(2^{\frac{C_{\mathrm{th}}}{\rho}}-1\right)}{\eta P_{u}(1-\rho) \beta^{2}}}\left[\frac{g_{s}}{\cos \left(\theta_{s}\right)}\right]^{\alpha_{\mathrm{us}}}}\left[\frac{K^{\prime}\left(\theta_{s}\right)}{K\left(\theta_{s}\right)+1}+\right. \\
& \left.\alpha_{\mathrm{us}}^{\prime} \ln \left(\frac{g_{s}}{\cos \left(\theta_{s}\right)}\right)+\alpha_{\mathrm{us}} \tan \left(\theta_{s}\right)\right]=\left(\frac{\mathcal{A}+2}{\mathcal{A}^{2}+2}\right) \frac{K^{\prime}\left(\theta_{s}\right)}{K\left(\theta_{s}\right)+1} .
\end{aligned}
$$

By denoting the solution to (B8) as $\theta_{s}^{\mathrm{c}}$, one have that $\left.\frac{\partial P_{\text {out }}}{\partial \theta_{s}}\right|_{\left(\theta_{s}=\theta_{s}^{\mathrm{c}}\right)}=0$. The feasible set of $\theta_{s}$ is $[0, \pi / 2]$. When $\theta_{s}=\pi / 2, \frac{g_{s}}{\cos \left(\theta_{s}\right)}$ and $\tan \left(\theta_{s}\right)$ go infinity, it leads to that the left side of (Bष]) is larger than the right side and $\left.\frac{\partial P_{\text {out }}}{\partial \theta_{s}}\right|_{\left(\theta_{s}=\frac{\pi}{2}\right)} \geq$ 0 . When $\theta_{s}=0, \tan \left(\theta_{s}\right)$ approaches 0 and $\alpha_{\mathrm{us}}^{\prime}$ is a small negative number, which leads to $\left.\frac{\partial P_{\text {out }}}{\partial \theta_{s}}\right|_{\left(\theta_{s}=0\right)} \leq\left.\frac{\partial P_{\text {out }}}{\partial \theta_{s}}\right|_{\left(\theta_{s}=\theta_{s}^{c}\right)} \leq 0$. Besides, $f_{1}=\frac{g_{s}}{\cos \left(\theta_{s}\right)}, f_{2}=\tan \left(\theta_{s}\right)$ and $f_{3}=\alpha_{\mathrm{us}}^{\prime}$ are increasing functions of $\theta_{s}$. In this case, although $f_{4}=\alpha_{\mathrm{us}}$ is a decreasing function of $\theta_{s}$, the decrement rate of $f_{4}$ is much smaller than 1 as the denominator of $f_{4}$ is larger than $1, f_{1}$ and $f_{2}$ increase from a relatively small value to $\infty$ as $\theta_{s}$ increases within $\left[0, \frac{\pi}{2}\right]$, so the decrement rate of $f_{4}$ is much slower than the increment rates of $f_{1}$ and $f_{2}$. As a result, the left side of (B8) is an increasing function of $\theta_{s}$. If $\theta_{s} \geq \theta_{s, c}$, then $\frac{\partial P_{\text {out }}}{\partial \theta_{s}} \geq 0$; otherwise, $\frac{\partial P_{\text {out }}}{\partial \theta_{s}} \leq 0$, which proves the pseudoconvexity of $P_{\text {out }}$. Additionally, the simulation results in Fig. $\square$ also implies that the outage probability is a pseudoconvex function of $h_{u}$.

\section{APPENDIX C \\ The Proof of LEMMA []}

The objective function in $\mathbf{P}_{\mathbf{A}}$ to be minimized is pseudoconvex in $\theta_{s}$, constraint $\mathrm{C}_{1}$ is affine, the optimal $\theta_{s}^{*}$ can be obtained by solving KKT conditions. With $\nu_{1}$ and $\nu_{2}$ being the Lagrange multipliers for constraints $\mathrm{C}_{1}$, the Lagrangian function of $\mathbf{P}_{\mathbf{A}}$ is given by

$$
\zeta_{\theta}\left(\nu_{1}, \nu_{2}, \theta_{s}\right)=P_{\text {out }}+\nu_{1}\left(\theta_{s}-\theta_{\max }\right)+\nu_{2}\left(\theta_{\min }-\theta_{s}\right) .
$$

The following KKT conditions are solved to find KKT points $\left(\theta_{s}^{*}, \nu_{1}^{*}, \nu_{2}^{*}\right)$,

$$
\left\{\begin{array}{l}
\nu_{1}^{*}, \quad \nu_{2}^{*} \geq 0 \\
\nu_{1}\left(\theta_{s}-\theta_{\max }\right)=0 \\
\nu_{2}\left(\theta_{\min }-\theta_{s}\right)=0 \\
\frac{\partial \zeta_{\theta}}{\partial \theta_{s}}=0
\end{array}\right.
$$

When $\nu_{1}=0$ and $\nu_{2}=0$, the critical point $\theta_{s, c}$ can be found in the implicit equation of (B8). When $\theta_{s, c} \geq \theta_{\max }$, one have that $\theta_{s}^{*}=\theta_{\max }$. And with $\mathrm{x}_{\max }=\mathrm{x}\left(\theta_{\max }\right), \mathrm{y}_{\max }=\mathrm{y}_{\mathrm{info}}\left(\theta_{\max }\right)$, $\mathrm{z}_{\max }=\mathrm{z}\left(\theta_{\max }\right)$, we have $\nu_{1, B}=-\frac{\partial P_{\mathrm{out}}\left(\theta_{\max }\right)}{\partial \theta_{s}}$, which is given by

$$
\begin{aligned}
& \nu_{1, B}=\left[\frac{2 Q_{1}\left(\mathrm{x}_{\max }, \mathcal{A} \mathrm{y}_{\max }\right) \mathrm{z}_{\max } \mathbf{K}_{\mathbf{0}}\left(\mathrm{z}_{\max }\right)}{Q_{1}\left(\mathrm{x}_{\max }, \mathrm{y}_{\max }\right)} \frac{\partial Q_{1}\left(\mathrm{x}_{\max }, \mathrm{y}_{\max }\right)}{\partial \theta_{s}}\right. \\
& \left.+\frac{\partial Q_{1}\left(\mathrm{x}_{\max }, \mathcal{A}_{\left.\mathrm{y}_{\max }\right)}\right)}{\partial \theta_{s}} \mathrm{z}_{\max } \mathbf{K}_{\mathbf{1}}\left(\mathrm{z}_{\max }\right)\right] .
\end{aligned}
$$

When $\theta_{s, c} \leq \theta_{\min }$, one has $\theta_{s}^{*}=\theta_{\min }$. As a result, we have $\nu_{2, B}=\frac{\partial P_{\text {out }}\left(\theta_{\min }\right)}{\partial \theta_{s}}$, which is given by

$$
\begin{aligned}
& \nu_{2, B}=-\left[\frac{2 Q_{1}\left(\mathrm{x}_{\min }, \mathcal{A} \mathrm{y}_{\min }\right) \mathrm{x}_{\min } \mathbf{K}_{\mathbf{0}}\left(\mathrm{z}_{\min }\right)}{Q_{1}\left(\mathrm{x}_{\min }, \mathrm{y}_{\min }\right)} \frac{\partial Q_{1}\left(\mathrm{x}_{\min }, \mathrm{y}_{\min }\right)}{\partial \theta_{s}}\right. \\
& \left.+\frac{\partial Q_{1}\left(\mathrm{x}_{\min }, \mathcal{A}_{\left.\mathrm{y}_{\min }\right)}\right)}{\partial \theta_{s}} \mathrm{Z}_{\min } \mathbf{K}_{\mathbf{1}}\left(\mathrm{z}_{\min }\right)\right] .
\end{aligned}
$$

As $P_{\text {out }}$ is pseudoconvex in $\theta_{s}$, there is a unique critical point $\theta_{s, c}$, which satisfies that

$$
\frac{\partial P_{\text {out }}}{\partial \theta_{s}} \begin{cases}\leq 0, & \text { if } \theta_{s} \leq \theta_{s, c} \\ \geq 0, & \text { if } \theta_{s, c} \leq \theta_{s}\end{cases}
$$

which matches the non-negativity of the Lagrange multipliers $\nu_{1, B}$ and $\nu_{2, B}$.

\section{APPENDIX D \\ The Proof of LEMMA 田 AND LEMMA}

Here, we prove the pseudo-convexity of the outage probability $P_{\text {out }}$ in $\rho$ with given $\theta_{s}$. Firstly, with $Q_{1}(a, b) \approx$ $\exp \left(-e^{\phi(a)} b^{\varphi(a)}\right)$, one has

$$
\left\{\begin{array}{l}
P_{\text {out }}^{(\mathrm{EH})}=1-\exp \left(-e^{\phi(x)} \mathcal{X}^{\mathcal{B}}\right), \\
P_{\text {out }}^{(\text {Info }}=1-\mathcal{G}_{1} \mathcal{G}_{2}^{\frac{\mathcal{B}}{2}} \mathbf{K}_{\mathbf{1}}\left(\mathcal{G}_{1} \mathcal{G}_{2}^{\frac{\mathcal{B}}{2}}\right) \\
P_{\text {out }}=1-\exp \left(-e^{\phi(x)} \mathcal{X}^{\mathcal{B}}\right) \mathcal{G}_{1} \mathcal{G}_{2}^{\frac{\mathcal{B}}{2}} \mathbf{K}_{\mathbf{1}}\left(\mathcal{G}_{1} \mathcal{G}_{2}^{\frac{\mathcal{B}}{2}}\right),
\end{array}\right.
$$

where $\mathcal{B}=\frac{\varphi(x)}{2}, \mathcal{X}=\left(\frac{2\left(K_{\mathrm{us}}+1\right) E_{\mathrm{th}} d_{\mathrm{us}}{ }^{\alpha} \mathrm{us}}{\eta P_{u} \beta(1-\rho) T}\right), \mathcal{G}_{1}=$ $2 e^{\phi(x)}\left(\frac{2\left(K_{\mathrm{us}}+1\right) d_{\mathrm{us}}{ }^{\alpha_{\mathrm{us}}}}{\beta}\right)^{\mathcal{B}}$ and $\mathcal{G}_{2}=\frac{N_{0} \rho\left(2^{\frac{C_{\mathrm{th}}}{\rho}}-1\right)}{\eta P_{u}(1-\rho)}$. By letting $\mathcal{G}=\mathcal{G}_{1} \mathcal{G}_{2}^{\frac{\mathcal{B}}{2}}$, the derivative of $P_{\text {out }}$ w.r.t $\rho$ is derived by

$$
\begin{aligned}
& -\frac{\partial P_{\text {out }}}{\partial \rho}= \\
& \frac{\partial \exp \left(-e^{\phi(x)} X^{\mathcal{B}}\right)}{\partial \rho} \mathcal{G} \mathbf{K}_{\mathbf{1}}(\mathcal{G})+\frac{\partial\left[\mathcal{G} \mathbf{K}_{\mathbf{1}}(\mathcal{G})\right]}{\partial \mathcal{G}} \frac{\partial[\mathcal{G}]}{\partial \rho} \exp \left(-e^{\phi(x)} X^{\mathcal{B}}\right) \\
& =\exp \left(-e^{\phi(x)} \mathcal{X}^{\mathcal{B}}\right)\left(-e^{\phi(x)} \mathcal{B} X^{\mathcal{B}-1} \frac{\partial X}{\partial \rho}\right) \mathcal{G} \mathbf{K}_{\mathbf{1}}(\mathcal{G})+ \\
& {\left[\mathbf{K}_{\mathbf{1}}(\mathcal{G})+\mathcal{G} \mathbf{K}_{\mathbf{1}}{ }^{\prime}(\mathcal{G})\right] \frac{\mathcal{B}}{2} \mathcal{G}_{1} \mathcal{G}_{2}^{\frac{\mathcal{B}}{2}-1} \frac{\partial\left[\mathcal{G}_{2}\right]}{\partial \rho} \exp \left(-e^{\phi(x)} X^{\mathcal{B}}\right) .}
\end{aligned}
$$$$
\text { Let } \frac{\partial P_{\text {out }}}{\partial \rho}=0 \text {, we have }
$$

$$
\begin{aligned}
& \left(-e^{\phi(x)} \mathcal{B} X^{\mathcal{B}-1} \frac{\partial X}{\partial \rho}\right) \mathcal{G} \mathbf{K}_{\mathbf{1}}(\mathcal{G})=\mathcal{G} \mathbf{K}_{\mathbf{0}}(\mathcal{G}) \frac{\mathcal{B}}{2} \mathcal{G}_{1} \mathcal{G}_{2}^{\frac{\mathcal{B}}{2}-1} \frac{\partial\left[\mathcal{G}_{2}\right]}{\partial \rho} \\
& \Rightarrow-\frac{\mathbf{K}_{\mathbf{1}}(\mathcal{G})}{\mathbf{K}_{\mathbf{0}}(\mathcal{G})} X^{\mathcal{B}-1} \frac{\partial X}{\partial \rho}=\left(\frac{2\left(K_{\mathrm{us}}+1\right) d_{\mathrm{us}}{ }^{\mathrm{us}}}{\beta}\right)^{\mathcal{B}} \mathcal{G}_{2}^{\frac{\mathcal{B}}{2}-1} \frac{\partial\left[\mathcal{G}_{2}\right]}{\partial \rho}
\end{aligned}
$$

With $\lim _{z \rightarrow \infty} \frac{\mathbf{K}_{\mathbf{1}}(z)}{\mathbf{K}_{\mathbf{0}}(z)}=1$, we have

$$
\left(\frac{E_{\mathrm{th}}}{\eta P_{u}(1-\rho)}\right)^{\mathcal{B}}=\mathcal{G}_{2}^{\frac{\mathcal{B}}{2}-1} \frac{N_{0}}{\eta P_{u}}\left(\frac{2^{\frac{C_{\mathrm{th}}}{\rho}} C_{\mathrm{th}} \ln 2(1-\rho)-\rho\left(2^{\frac{C_{\mathrm{th}}}{\rho}}-1\right)}{\rho(1-\rho)}\right),
$$

where the solution of (45) is denoted as $\rho_{c}$. It is noticed that $\rho(1-\rho)\left(\frac{E_{\mathrm{th}}}{\eta P_{u}(1-\rho)}\right)^{\mathcal{B}}$ is an increasing function of $\rho$, and 
$\mathcal{G}_{2}^{\frac{\mathcal{B}}{2}-1}\left(C_{\text {th }} \ln 2(1-\rho)-\rho\right)\left(\left(2^{\frac{C_{\text {th }}}{\rho}}-1\right)\right)+C_{\text {th }} \ln 2(1-\rho)$ decreases with $\rho$. If $\rho \geq \rho_{c}$, then $\frac{\partial P_{\text {out }}}{\partial \rho} \geq 0$, otherwise if $\rho \leq \rho_{c}$, then $\frac{\partial P_{\text {out }}}{\partial \rho} \leq 0$. As unimodality of a single variable function is equivalent to its pseudoconvexity, the pseudoconvexity of $P_{\text {out }}$ in $\rho$ has been proved. At the same, the simulation in Fig. 3 also implies that the outage probability is a pseudoconvex function of $\rho$.

For the special case that energy threshold is small enough, the energy-constrained outage probability can be ignored, i.e.,

$$
P_{\text {out }}^{(\mathrm{EH})}=1-Q_{1}\left(\sqrt{2 K_{\mathrm{us}}}, \sqrt{\frac{2\left(K_{\mathrm{us}}+1\right) E_{\mathrm{th}} d_{\mathrm{us}}{ }^{\alpha_{\mathrm{us}}}}{\eta P_{u} \beta(1-\rho) T}}\right)=0,
$$

where the energy threshold obtained by solving $P_{\mathrm{out}}^{(\mathrm{EH})}=0$ is $E_{\mathrm{th}}=\frac{\eta P_{u} \beta_{0}(1-\rho)\left[Q_{1}^{-1}\left(\sqrt{2 K_{\mathrm{us}}}, 1\right)\right]}{2\left(K_{\mathrm{us}}+1\right) d_{\mathrm{us}}{ }^{\alpha_{\mathrm{us}}}}$. And then, the optimal TS factor $\rho_{\text {info for minimizing the rate-constrained outage }}^{*}$ probability is obtained by solving $\frac{\partial P_{\text {out }}^{(\text {Inf })}}{\partial \rho}=0$, i.e.,

$$
\left\{\begin{array}{l}
\frac{\partial P_{\text {out }}^{(\text {Inf })}}{\partial \rho}=\frac{\partial\left[\mathcal{G} \mathbf{K}_{\mathbf{1}}(\mathcal{G})\right]}{\partial \mathcal{G}} \frac{\partial[\mathcal{G}]}{\partial \rho}=0, \\
{\left[\mathbf{K}_{\mathbf{1}}(\mathcal{G})+\mathcal{G} \mathbf{K}_{\mathbf{1}}{ }^{\prime}(\mathcal{G})\right] \frac{\mathcal{B}}{2} \mathcal{G}_{1} \mathcal{G}_{2}^{\frac{\mathcal{B}}{2}-1} \frac{\partial\left[\mathcal{G}_{2}\right]}{\partial \rho}=0,} \\
\frac{2^{\frac{C_{\text {th }}}{\rho}} C_{\text {th }} \ln 2(1-\rho)-\rho\left(2^{\frac{C_{\text {th }}}{\rho}}-1\right)}{\left[\frac{\mathcal{B}}{2} \mathcal{G}_{1} \mathcal{G}_{2}^{\frac{\mathcal{B}}{2}-1}\right]^{-1}\left[\mathcal{G} \mathbf{K}_{\mathbf{0}}(\mathcal{G})\right]^{-1} \rho(1-\rho)^{2}}=0 .
\end{array}\right.
$$

The solution of (46d) is

$$
\rho_{\text {info }}=\frac{C_{\mathrm{th}} \ln 2}{1+C_{\mathrm{th}} \ln 2+\mathcal{W}\left(-e^{-C_{\mathrm{th}} \ln 2-1}\right)},
$$

where $\mathcal{W}(\chi)$ is the Lambert $\mathcal{W}$ function with $\mathcal{W}(\chi) e^{\mathcal{W}(\chi)}=$ $\chi$. Thus, the proof for Lemma $[$ is completed.

\section{APPENDIX E}

\section{THE PROOF OF LEMMA 6}

On the coverage boundary of the UAV, the outage probability satisfies that $P_{\text {out }}=P_{\mathrm{th}}$, and its derivative w.r.t x yields

$$
\begin{aligned}
& -\frac{\partial P_{\text {out }}}{\partial \mathrm{x}} \\
& =Q_{1}\left(\mathrm{x}, \mathcal{A} \mathrm{y}_{\text {info }}\right) \frac{\partial\left[z \mathbf{K}_{\mathbf{1}}(z)\right]}{\partial z} \frac{\partial[z]}{\partial u} \frac{\partial[u]}{\partial \mathrm{x}}+\frac{\partial Q_{1}\left(\mathrm{x}, \mathcal{A} \mathrm{y}_{\text {info }}\right)}{\partial \mathrm{x}} z \mathbf{K}_{\mathbf{1}}(z) .
\end{aligned}
$$

By letting $\frac{\partial P_{\text {out }}}{\partial \mathrm{x}}=0$, with $\lim _{z \rightarrow \infty} \frac{\mathbf{K}_{1}(z)}{\mathbf{K}_{\mathbf{0}}(z)}=1$ and $\frac{\partial Q_{1}(x, y)}{\partial x}=$ $\frac{\partial Q_{1}(x, y)}{\partial x}+\frac{\partial Q_{1}(x, y)}{\partial y} \frac{\partial y}{\partial x}$, we have

$$
\frac{\partial \mathrm{y}_{\text {info }}}{\partial \mathrm{x}}=\left(\frac{\mathcal{A}}{\mathcal{A}^{2}+2} \frac{I_{1}\left(\mathrm{x} \cdot \mathrm{y}_{\text {info }}\right)}{I_{0}\left(\mathrm{x} \cdot \mathrm{y}_{\text {info }}\right)}+\frac{2}{\mathcal{A}^{2}+2} \frac{I_{1}\left(\mathrm{xy}_{\text {info }}\right)}{I_{0}\left(\mathrm{xy}_{\text {info }}\right)}\right)
$$

where the derivation process is similar to the proof for Lemma $\square$ in Appendix [B]. Based on [B]], for small $x$, we have

$$
I_{n}(x y) \approx\left(\frac{x y}{2}\right)^{n}, n=\{0,1\}
$$

Then (48) is rewritten as $\frac{\partial \mathrm{y}_{\text {info }}}{\partial \mathrm{x}}=\left(\frac{\mathcal{A}+2}{\mathcal{A}^{2}+2}\right) \frac{\mathrm{xy}_{\text {info }}}{2}$, which is the first order differential equation with the solution of

$$
\mathrm{y}_{\text {info }}=\mathrm{y}_{0} e^{\frac{\left(\frac{\mathcal{A}+2}{\mathcal{A}^{2}+2}\right) \mathrm{x}^{2}}{4}},
$$

where $\mathrm{y}_{0}$ is the value of $\mathrm{y}_{\text {info }}$ at $\mathrm{x}=0$. And $\mathrm{y}_{0}$ can be obtained from the following equation

$1+2 Q_{1}\left(0, \mathcal{A y}_{0}\right) \ln \left(Q_{1}\left(0, \mathrm{y}_{0}\right)\right) \mathbf{K}_{1}\left(-2 \ln \left(Q_{1}\left(0, \mathrm{y}_{0}\right)\right)\right)=P_{\mathrm{th}}$.
From [ [26], one sees that $Q_{1}\left(0, \mathrm{y}_{0}\right)=e^{-\frac{\mathrm{y}_{0}^{2}}{2}}$, and then ([1]) is simplified to

$$
e^{-\frac{\left(\mathcal{A}_{0}\right)^{2}}{2}} \mathrm{y}_{0}^{2} \mathbf{K}_{\mathbf{1}}\left(\mathrm{y}_{0}^{2}\right)=1-P_{\mathrm{th}} .
$$

When $\mathrm{x}$ is large, according to $(\overline{B 6})$, the (48) is rewritten as

$$
\frac{\partial \mathrm{y}_{\text {info }}}{\partial \mathrm{x}}=\frac{\mathcal{A}}{\mathcal{A}^{2}+2}\left(1-\frac{1}{2 \mathrm{x} \mathrm{y}_{\text {info }}}\right)+\frac{2}{\mathcal{A}^{2}+2}\left(1-\frac{1}{2 \mathrm{xy}_{\text {info }}}\right) \approx \frac{\mathcal{A}+2}{\mathcal{A}^{2}+2} .
$$

Thus, one has

$$
\mathrm{y}_{\text {info }}=\left(\frac{\mathcal{A}+2}{\mathcal{A}^{2}+2}\right) \mathrm{x}+c_{1} .
$$

By bring (54) into (53), one has

$$
\frac{\partial \mathrm{y}_{\text {info }}}{\partial \mathrm{x}}=\frac{\mathcal{A}+2}{\mathcal{A}^{2}+2}-\frac{3(\mathcal{A}+2)}{2\left(\mathcal{A}^{2}+2\right) c_{1}}\left(\frac{1}{(\mathcal{A}+2) \mathrm{x}}-\frac{1}{(\mathcal{A}+2) \mathrm{x}+\left(\mathcal{A}^{2}+2\right) c_{1}}\right),
$$

Therefore, taking the integral of the above equation obtains

$\mathrm{y}_{\text {info }}=\left(\frac{\mathcal{A}+2}{\mathcal{A}^{2}+2}\right) \mathrm{x}-\frac{3}{2\left(\mathcal{A}^{2}+2\right) c_{1}}\left[\ln \left(\frac{\mathrm{x}}{(\mathcal{A}+2) \mathrm{x}+\left(\mathcal{A}^{2}+2\right) c_{1}}\right)\right]+c_{2}$.

It is noted that as $\mathrm{x} \rightarrow \infty$, from (55) we have that $\mathrm{y}_{\text {info }}=$ $\left(\frac{\mathcal{A}+2}{\mathcal{A}^{2}+2}\right) \mathrm{x}+c_{2}$. Combing with (54), one can obtain $c_{1}=c_{2} \triangleq c$. When $c=0$, we re-compute $\mathrm{y}_{\text {info }}$ by bringing $\mathrm{y}_{\text {info }}=$ $\left(\frac{\mathcal{A}+2}{\mathcal{A}^{2}+2}\right) \mathrm{x}$ into (153). Thus, we have $\frac{\partial \mathrm{y}_{\text {info }}}{\partial \mathrm{x}}=\frac{\mathcal{A}+2}{\mathcal{A}^{2}+2}-\frac{3}{2(\mathcal{A}+2) \mathrm{x}^{2}}$, which has the solution of

$$
\mathrm{y}_{\text {info }}=\left(\frac{\mathcal{A}+2}{\mathcal{A}^{2}+2}\right) \mathrm{x}+\frac{3}{2(\mathcal{A}+2) \mathrm{x}} .
$$

Consequently, the relationship between $\mathrm{y}_{\text {info }}$ and $\mathrm{x}$ is summarized as

$$
\mathrm{y}_{\text {info }}=\left\{\begin{array}{l}
\mathrm{y}_{0} e^{\frac{\left(\frac{\mathcal{A}+2}{\mathcal{A}^{2}+2}\right) \mathrm{x}^{2}}{4}}, \mathrm{x} \leq \mathrm{x}_{\mathrm{c}} \\
\left(\frac{\mathcal{A}+2}{\mathcal{A}^{2}+2}\right) \mathrm{x}-\frac{3 \ln \left(\frac{\mathrm{x}}{(\mathcal{A}+2) \mathrm{x}+\left(\mathcal{A}^{2}+2\right) c}\right)}{2\left(\mathcal{A}^{2}+2\right) c}+c, \\
\mathrm{x} \geq \mathrm{x}_{\mathrm{c}} \wedge c \neq 0 \\
\left(\frac{\mathcal{A}+2}{\mathcal{A}^{2}+2}\right) \mathrm{x}+\frac{3}{2(\mathcal{A}+2) \mathrm{x}}, \mathrm{x} \geq \mathrm{x}_{\mathrm{c}} \wedge c=0
\end{array}\right.
$$

where $\mathrm{x}_{\mathrm{c}}$ is the intersection of the sub-functions. For $x \gg 1$, which results in $x \geq x_{c}$, it can be seen that $\frac{3 \ln \left(\frac{x}{(\mathcal{A}+2) x+\left(\mathcal{A}^{2}+2\right) c}\right)}{2\left(\mathcal{A}^{2}+2\right) c}$ and $\frac{3}{2(\mathcal{A}+2) x}$ can be neglected, and then we have

$$
\mathrm{y}_{\text {info }} \approx\left(\frac{\mathcal{A}+2}{\mathcal{A}^{2}+2}\right) \mathrm{x}+c .
$$

In order to determine $c$, one can find that $x \rightarrow \infty$ results in $\mathrm{y}_{\text {info }}=\left(\frac{\mathcal{A}+2}{\mathcal{A}^{2}+2}\right) \mathrm{x}+c \rightarrow \infty$, and $y \gg y-\left(\frac{\mathcal{A}+2}{\mathcal{A}^{2}+2}\right) x$. By using the asymptotic relationship between the generalized Marcum $Q$-function and the Gaussian $Q$-function [35], one can have that $Q_{1}(\mathrm{x}, \mathrm{y}) \approx Q\left(y-\left(\frac{\mathcal{A}+2}{\mathcal{A}^{2}+2}\right) x\right) \approx Q(c)$, then we have

$$
1+2 Q(c) \log (Q(c)) \mathbf{K}_{\mathbf{1}}(-2 \log (Q(c)))=\mathrm{P}_{\mathrm{th}} .
$$

Thereby, $c$ can be obtained by solving (58).

\section{APPENDIX F}

\section{THE Proof OF LEMMA $\square$}

By using the relationship between $\mathrm{x}$ and $\mathrm{y}_{\text {info }}$ shown in Lemma 6 , we aim to find the critical point of $R_{\mathrm{us}}$ by solving 
$\frac{\partial R_{\mathrm{us}}}{\partial \theta_{\mathrm{s}}}=0$, and the derivative of $\ln \left(R_{\mathrm{us}}\right)$ is given by

$$
\begin{aligned}
& \left.\frac{\partial \ln \left(R_{\mathrm{us}}\left(\theta_{s}\right)\right)}{\partial \theta_{s}}=\frac{\partial \ln \left[\left(\frac{\beta \mathrm{y}_{\mathrm{info}}{ }^{2}}{\left(x^{2}+2\right) \sqrt{\mathcal{G}_{2}}}\right)^{\frac{1}{\alpha_{\mathrm{us}}}} \cos \left(\theta_{s}\right)\right]}{\partial \theta_{s}}\right]-\tan \left(\theta_{s}\right) \\
& =\frac{\partial}{\partial \theta_{s}}\left[\frac{\ln \left(\frac{\beta}{\sqrt{\mathcal{G}_{2}}}\right)+2 \ln \left(\left(\frac{\mathcal{A}+2}{\mathcal{A}^{2}+2}\right) x+c\right)-\ln \left(x^{2}+2\right)}{\alpha_{\mathrm{us}}}\right] \\
& =\frac{2 x^{\prime}\left(\frac{\mathcal{A}+2}{(\mathcal{A}+2) x+\left(\mathcal{A}^{2}+2\right) c}-\frac{x}{x^{2}+2}\right) \alpha_{\mathrm{us}}-\alpha_{\mathrm{us}}^{\prime} \alpha_{\mathrm{us}} \ln \left(d_{\mathrm{us}}\right)}{\alpha_{\mathrm{us}}^{2}}-\tan \left(\theta_{s}\right) .
\end{aligned}
$$

By letting $\frac{\partial \ln \left(R_{\mathrm{us}}\left(\theta_{s}\right)\right)}{\partial \theta_{s}}=0$, the optimal $\theta_{s}$ to maximize the coverage radius can be found in the following equation

$$
\begin{aligned}
& 2 x^{\prime}\left(\frac{\mathcal{A}+2}{(\mathcal{A}+2) x+\left(\mathcal{A}^{2}+2\right) c}-\frac{x}{x^{2}+2}\right) \\
& =\alpha_{\mathrm{us}} \tan \left(\theta_{s}\right)+\alpha_{\mathrm{us}}^{\prime}\left(\theta_{s}\right) \ln \left(d_{\mathrm{us}}\left(\theta_{s}\right)\right) .
\end{aligned}
$$

where the solution of (5प) is denoted as $\theta_{s, c}$, i.e., $\left.\frac{\partial \ln \left(R_{\mathrm{us}}\right)}{\partial \theta_{s}}\right|_{\left(\theta_{s}=\theta_{s, c}\right)}=0$. The feasible set of $\theta_{s}$ is $[0, \pi / 2]$. When $\theta_{s}=\pi / 2, \tan \left(\theta_{s}\right)$ goes infinity, which leads to that the right side of (5Q) is larger than left side and $\frac{\partial \ln \left(R_{\mathrm{us}}\right)}{\partial \theta_{s}} \leq 0$. And then when $\theta_{s}=0$, the right side of (5耳) takes the minimum value, which leads to $\left.\frac{\partial \ln \left(R_{\mathrm{us}}\right)}{\partial \theta_{s}}\right|_{\left(\theta_{s}=0\right)} \geq\left.\frac{\partial \ln \left(R_{\mathrm{us}}\right)}{\partial \theta_{s}}\right|_{\left(\theta_{s}=\theta_{s, c}\right)} \geq 0$. It is noticed that the right side of (5Y) is an increasing function and the left side of (59) is a decreasing function, therefore, one have that if $\theta_{s} \geq \theta_{s, c}$, then $\frac{\partial \ln \left(R_{\mathrm{us}}\right)}{\partial \theta_{s}} \leq 0$; otherwise, if $\theta_{s} \leq \theta_{s, c}$, then $\frac{\partial \ln \left(R_{\mathrm{us}}\right)}{\partial \theta_{s}} \geq 0$, which proves the pseudoconcavity of $\ln \left(R_{\mathrm{us}}\right)$ in $\theta_{s}$.

With $\lambda_{1}$ and $\lambda_{2}$ being the Lagrange multipliers for the maximum and minimum elevation angle constraints, the Lagrangian function of problem $\mathbf{P}_{\mathbf{B}}$ is given by

$$
\zeta_{B}\left(\lambda_{1}, \lambda_{2}, \theta_{s}\right)=\ln \left(R_{\mathrm{us}}\right)-\lambda_{1}\left(\theta_{s}-\theta_{\max }\right)-\lambda_{2}\left(\theta_{\min }-\theta_{s}\right) .
$$

The following KKT conditions are solved to find KKT points $\left(\theta_{s}^{*}, \lambda_{1}^{*}, \lambda_{2}^{*}\right)$

$$
\left\{\begin{array}{l}
\lambda_{1}^{*}, \quad \lambda_{2}^{*} \geq 0, \\
\lambda_{1}\left(\theta_{s}-\theta_{\max }\right)=0, \\
\lambda_{2}\left(\theta_{\min }-\theta_{s}\right)=0, \\
\frac{\partial \zeta_{B}}{\partial \theta_{s}^{*}}=0 .
\end{array}\right.
$$

When $\lambda_{1}=0$ and $\lambda_{2}=0$, the critical point $\theta_{s, c}$ to maximize the coverage radius can be obtained by solving $\frac{\partial \ln \left(R_{\mathrm{us}}\right)}{\theta_{s}}=0$, which is given in (59). When $\theta_{s, c} \geq \theta_{\max }$, one have that $\theta_{s}^{*}=$ $\theta_{\max }$ and $\lambda_{1}=\frac{\partial \ln \left(\left(R_{\text {us }}\left(\theta_{\max }\right)\right)\right.}{\partial \theta_{s}}$. When $\theta_{s, c} \leq \theta_{\min }$, one have that $\theta_{s}^{*}=\theta_{\min }$. and $\lambda_{2}=-\frac{\partial \ln \left(R_{\mathrm{us}}\left(\theta_{\min }\right)\right)}{\partial \theta_{s}}$. As $\ln \left(R_{\mathrm{us}}\right)$ is a pseudoconcave function in $\theta_{s}$, there is a unique critical point $\theta_{s, c}$, which satisfies that

$$
\frac{\partial \ln \left(R_{\mathrm{us}}\right)}{\partial \theta_{s}} \begin{cases}\geq 0, & \text { if } \theta_{s} \leq \theta_{s, c} \\ \leq 0, & \text { if } \theta_{s, c} \leq \theta_{s}\end{cases}
$$

which matches the non-negativity of the Lagrange multipliers $\lambda_{1}$ and $\lambda_{2}$. The proof of Lemma 7 is completed.

\section{REFERENCES}

[1] Y. Zeng, R. Zhang and T. J. Lim, "Wireless communications with unmanned aerial vehicles: opportunities and challenges," in IEEE Commun. Mag., vol. 54, no. 5, pp. 36-42, May 2016.
[2] M. T. Dabiri, H. Safi, S. Parsaeefard and W. Saad, "Analytical channel models for millimeter wave UAV networks under hovering fluctuations," IEEE Trans. Wirel. Commun., vol. 19, no. 4, pp. 2868-2883, Apr. 2020.

[3] Y. Zeng, J. Xu, R. Zhang, "Energy minimization for wireless communication with rotary-wing UAV," IEEE Trans. Wirel. Commun., vol. 18, no. 4, pp. 2329-2345, Apr. 2019.

[4] H. Hu, K. Xiong, G. Qu, Q. Ni, P. Fan and K. B. Letaief, "AoI-minimal trajectory planning and data collection in UAV-assisted wireless powered IoT networks," IEEE Internet Things J., vol. 8, no. 2, pp. 1211-1223, Jan. 2021.

[5] R. H. Jiang, K. Xiong, P. Y. Fan, Y. Zhang, Z. D. Zhong, "Power minimization in SWIPT networks with coexisting power-splitting and time-switching users under nonlinear EH model," IEEE Internet Things J., vol. 6, no. 5, PP. 8853-8869, Oct. 2019.

[6] K. Xiong, P. Fan, C. Zhang and K. B. Letaief, "Wireless information and energy transfer for two-hop non-regenerative MIMO-OFDM relay networks," IEEE J. Sel. Areas Commun., vol. 33, no. 8, pp. 1595-1611, Aug. 2015.

[7] Y. Hu, X. Yuan, J. Xu and A. Schmeink, "Optimal 1D trajectory design for UAV-enabled multiuser wireless power transfer," IEEE Trans. Commun., vol. 67, no. 8, pp. 5674-5688, Aug. 2019.

[8] X. Di, K. Xiong, P. Fan and H. Yang, "Simultaneous wireless information and power transfer in cooperative relay networks with rateless codes," IEEE Trans. Veh. Tech., vol. 66, no. 4, pp. 2981-2996, Apr. 2017.

[9] Y. Lu, K. Xiong, P. Y. Fan, Z. D. Zhong, K. B. Letaief, "Robust transmit beamforming with artificial redundant signals for secure SWIPT system under non-linear EH model," IEEE Trans. Wirel. Commun., vol. 17, no. 4, pp. 2218-2232, Apr. 2018.

[10] Q. Q. W, Y. Zeng, and R. Zhang, "Joint trajectory and communication design for multi-UAV enabled wireless networks," IEEE Trans. Wirel. Commun., vol. 17, no. 3, pp. 2109-2121, Mar. 2018.

[11] X. Wang and M. C. Gursoy, "Coverage analysis for energy-harvesting UAV-assisted mmWave cellular networks," IEEE J. Sel. Areas Commun., vol. 37, no. 12, pp. 2832-2850, Dec. 2019.

[12] L. Xiao, Y. Xu, D. Yang and Y. Zeng, "Secrecy energy efficiency maximization for UAV-enabled mobile relaying," IEEE Trans. Green Commun. \& Network., vol. 4, no. 1, pp. 180-193, Mar 2020.

[13] Y. Liu, K. Xiong, Q. Ni, P. Fan and K. B. Letaief, "UAV-assisted wireless powered cooperative mobile edge computing: joint offloading, CPU control, and trajectory optimization," IEEE Internet of Things J., vol. 7, no. 4, pp. 2777-2790, Apr. 2020.

[14] X. Y. Hu, K. Wong, K. Yang, Z. B. Zheng, "UAV-assisted relaying and edge computing: scheduling and trajectory optimization," IEEE Trans. Wirel. Commun., vol. 18, no. 10, pp. 4738-4752, Oct. 2019.

[15] Y. Zhou, C.H. Pan et al, "Secure communications for UAV-enabled mobile edge computing systems," IEEE Trans. Commun., vol. 68, no. 1, pp. 376-388, Jan. 2020.

[16] J. Xu, Y. Zeng, R. Zhang, "UAV-enabled wireless power transfer: trajectory design and energy optimization," IEEE Trans. Wirel. Commun., vol. 17, no. 8, pp. 5092-5106, Aug. 2018.

[17] D. N. K. Jayakody, T. D. P. Perera, A. Ghrayeb and M. O. Hasna, "Selfenergized UAV-assisted scheme for cooperative wireless relay networks," IEEE Trans. Veh. Technol., vol. 69, no. 1, pp. 578-592, Jan. 2020.

[18] X. Fu, T. Ding, M. Kadoch and M. Cheriet, "Uplink performance analysis of UAV cellular communications with power control," in Proc. IWCMC, pp. 676-679, 2020.

[19] P. Lohan and D. Mishra, "Utility-aware optimal resource allocation protocol for UAV-assisted small cells with heterogeneous coverage demands," IEEE Trans. Wirel. Commun., vol. 19, no. 2, pp. 1221-1236, Feb. 2020.

[20] T. Shen and H. Ochiai, "UAV-aided data collection for wireless powered sensor network over Rician fading channels," in Proc. IEEE CCNC, pp. $1-5,2019$.

[21] H. Tu, J. Zhu and Y. Zou, "Optimal power allocation for minimizing outage probability of UAV relay communications," in Proc. WCSP, pp. 1-6, 2019.

[22] M. M. Azari, F. Rosas, K. Chen and S. Pollin, "Optimal UAV positioning for terrestrial-aerial communication in presence of fading," Proc. IEEE GC, pp. 1-7, Dec. 2018.

[23] C. You and R. Zhang, "3D Trajectory optimization in rician fading for UAV-enabled data harvesting," IEEE Trans. Wirel. Commun., vol. 18, no. 6, pp. 3192-3207, Jun. 2019.

[24] M. M. Azari, F. Rosas, K. Chen and S. Pollin, "Ultra reliable UAV communication using altitude and cooperation diversity," IEEE Trans. Wirel. Commun., vol. 66, no. 1, pp. 330-344, Jan. 2018.

[25] R. Amorim, H. Nguyen, P. Mogensen, et al, "Radio channel modeling for UAV communication over cellular networks," IEEE Commun. Lett., vol. 6 , no. 4, pp. 514-517, Aug. 2017. 
[26] R. T. Short, "Computation of Rice and noncentral chi-squared probabilities," PhaseLocked Syst., Ottawa, ON, Canada, Tech. Rep. PHS0254, 2012.

[27] A. Al-Hourani, S. Kandeepan and S. Lardner, "Optimal lap altitude for maximum coverage," IEEE Commun. Lett., vol. 3, no. 6, pp. 569-572, Dec. 2014.

[28] C. You and R. Zhang, "Hybrid offline-online design for UAV-enabled data harvesting in probabilistic LoS channels," IEEE Trans. Wirel. Commun., vol. 19, no. 6, pp. 3753-3768, June 2020.

[29] J. Hagenauer, et al., "The maritime satellite communication channelchannel model, performance of modulation and coding," IEEE J. Sel. Areas Commun., vol. 5, no. 4, pp. 701-713, May 1987.

[30] M. Z. Bocus, C. P. Dettmann and J. P. Coon, "An approximation of the first order Marcum Q-function with application to network connectivity analysis," IEEE Commun. Lett., vol. 17, no. 3, pp. 499-502, Mar. 2013.

[31] D. Mishra and S. De, "i2RES: Integrated information relay and energy supply assisted RF harvesting communication," IEEE Trans. Commun., vol. 65, no. 3, pp. 1274-1288, Mar. 2017.

[32] A. Cambini and L. Martein, Generalized Convexity and Optimization: Theory and Applications, Berlin, Heidelberg: Springer-Verlag, 2009.

[33] M. S. Bazaraa, H. D. Sherali, and C. M. Shetty, Nonlinear Programming: Theory and Applications. New York, NY, USA: Wiley, 2006

[34] A. D. Belegundu and T. R. Chandrupatla, Optimization Concepts and Applications in Engineering. Cambridge, U.K.: Cambridge Univ. Press, 2011.

[35] F. J. Lopez-Martinez, et al., "Asymptotically exact approximations for the symmetric difference of generalized Marcum $Q$-functions," IEEE Trans. Veh. Technol., vol. 64, no. 5, pp. 2154-2159, May 2015.

[36] R. Vazquez and M. Krstic, "Marcum Q-functions and explicit feedback laws for stabilization of constant coefficient $2 \times 2$ linear hyperbolic systems," in Proc. Decision and Control, pp. 466-471, 2013.

[37] J. Segura, "Bounds for ratios of modified Bessel functions and associated turán-type inequalities," J. Math. Anal. Appl., vol. 374, no. 2, pp. 516528, 2011.

[38] M. Avriel, W. E. Diewert, S. Schaible, and I. Zang, Generalized Concavity, vol. 63. Philadelphia, PA, USA: SIAM, 2010.

[39] I. Csiszár and G. Tusnády, "Information geometry and alternating minimization procedures," Statist. Decisions, vol. 1, no. 1, pp. 205-237, 1984.

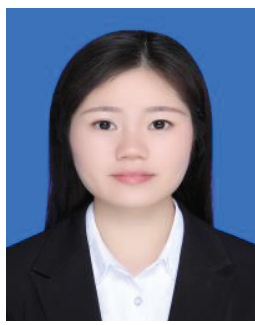

Yuan Liu received the B.S. degree from College of Computer and Information Technology, Liaoning Normal University, Dalian, China, in 2017. She is currently pursuing the $\mathrm{Ph} . \mathrm{D}$. degree with the School of Computer and Information Technology, Beijing Jiaotong University, Beijing, China. Her current research interests include UAV communications, energy harvesting in wireless communication networks, wireless sensor networks.

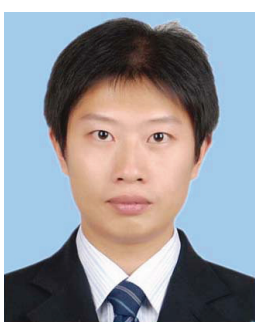

Ke Xiong received the B.S. and Ph.D. degrees from Beijing Jiaotong University (BJTU), Beijing, China, in 2004 and 2010, respectively.

From April 2010 to February 2013, he was a PostDoctoral Research Fellow with the Department of Electronics Engineering, Tsinghua University, Beijing. Since March 2013, he has been a Lecturer, an Associate Professor of BJTU, where he is currently a Full Professor and the Vice Dean of the School of Computer and Information Technology. From September 2015 to September 2016, he was a Visiting Scholar with the University of Maryland, College Park, MD, USA. He has published more than 100 academic papers in referred journals and conferences. His current research interests include wireless cooperative networks, wireless powered networks, and network information theory. He is a member of China Computer Federation (CCF) and also a senior member of the Chinese Institute of Electronics (CIE). He serves as the Associate Editor-in Chief for the Chinese Journal New Industrialization Strategy, and an Editor of Computer Engineering and Software. In 2017, he serves as the leading Editor for the Special Issue Recent Advances in Wireless Powered Communication Networks for EURASIP Journal on Wireless Communications and Networking and the Guest Editor of the Special Issue Recent Advances in Cloud-Aware Mobile Fog Computing for Wireless Communications and Mobile Computing. He currently also serves as a Reviewer for more than 15 international journals, including IEEE TRANSACTIONS ON SIGNAL PROCESSING, IEEE TRANSACTIONS ON WIRELESS COMMUNICATIONS, IEEE TRANSACTIONS ON COMMUNICATIONS, IEEE TRANSACTIONS ON VEHICULAR TECHNOLOGY, IEEE COMMUNICATIONS LETTERS, IEEE SIGNAL PROCESSING LETTERS, and IEEE WIRELESS COMMUNICATION LETTERS. He also served as the Session Chair for IEEE GLOBECOM'2012, IET ICWMMN'2013, IEEE ICC'2013, ACM MOMM'2014, and the Publicity and the Publication Chair for IEEE HMWC'2014, as well as the TPC Co-Chair for IET ICWMMN'2017 and IET ICWMMN'2019. He won the best student paper awards of the HWMC'2014, the best student paper awards of the 25th and 26th Annual Conference of Information Theory of CIT (CIT-IT), the IEEE ICC'2020 and also the TAOS Technical Committee at IEEE ICC'2020.

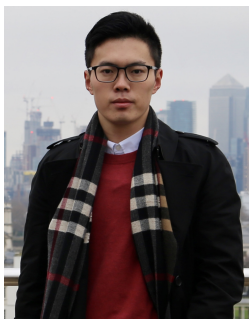

Yang Lu received the B.E. and Ph.D. degrees from Beijing Jiaotong University (BJTU), Beijing, China, in 2014 and 2020, respectively. Since 2020, he has been with BJTU as a Professor in computer and information technology. From Feb. 2016 to Aug. 2016 \& Mar. 2018 to July 2018, he was a visiting scholar at National Tsing Hua University. From July 2017 to Jan. 2018, he was a visiting scholar at Lancaster University. From Oct. 2018 to Oct. 2019, he was a visiting scholar at University of Florida. His current research interests include the energy efficient communication system design and SWIPT. He serves as a reviewer for IEEE Journal on Selected Areas in Communications, IEEE Transactions on Wireless Communications, IEEE Transactions on Communications, IEEE Transactions on Vehicular Technology and IEEE Wireless Communications Letters. 


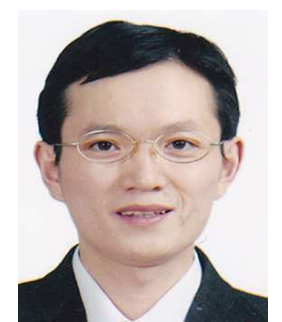

Qiang Ni (M'04-SM'08) received the B.Sc., M.Sc., and $\mathrm{Ph} . \mathrm{D}$. degrees from the Huazhong University of Science and Technology, China, all in engineering. $\mathrm{He}$ is currently a Professor and the Head of the Communication Systems Group, School of Computing and Communications, Lancaster University, Lancaster, U.K. His research interests include the area of future generation communications and networking, including green communications and networking, millimeter-wave wireless communications, cognitive radio network systems, non-orthogonal multiple access (NOMA), heterogeneous networks, 5G and 6G, SDN, cloud networks, energy harvesting, wireless information and power transfer, IoTs, cyber physical systems, AI and machine learning, big data analytics, and vehicular networks. He has authored or co-authored $300+$ papers in these areas. $\mathrm{He}$ was an IEEE 802.11 Wireless Standard Working Group Voting Member and a contributor to various IEEE wireless standards.

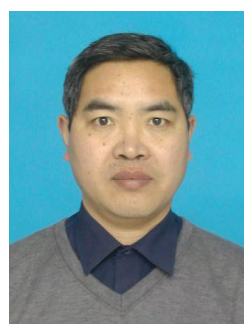

Pingyi Fan (M'03-SM'09) received the B.S and M.S. degrees from the Department of Mathematics of Hebei University in 1985 and Nankai University in 1990, respectively, received his Ph.D. degree from the Department of Electronic Engineering, Tsinghua University, Beijing, China in 1994. He is a professor of department of EE of Tsinghua University currently. From Aug. 1997 to March. 1998, he visited Hong Kong University of Science and Technology as Research Associate. From May. 1998 to Oct. 1999, he visited University of Delaware, USA, as research fellow. In March. 2005, he visited NICT of Japan as visiting Professor. From June. 2005 to May 2014, he visited Hong Kong University of Science and Technology for many times and from July 2011 to Sept. 2011, he is a visiting professor of Institute of Network Coding, Chinese University of Hong Kong.

Dr. Fan is a senior member of IEEE and an oversea member of IEICE. He has attended to organize many international conferences including as General co-Chair of EAI Chinacom2020, and IEEE VTS HMWC2014, TPC co-Chair of IEEE International Conference on Wireless Communications, Networking and Information Security (WCNIS 2010) and TPC member of IEEE ICC, Globecom, WCNC, VTC, Inforcom etc. He has served as an editor of IEEE Transactions on Wireless Communications, Inderscience International Journal of Ad Hoc and Ubiquitous Computing, Wiley Journal of Wireless Communication and Mobile Computing, MDPI Electronics, and Open Journal of Mathematical Sciences etc. He is also a reviewer of more than 30 international Journals including 20 IEEE Journals and 8 EURASIP Journals. $\mathrm{He}$ has received some academic awards, including the IEEE WCNC'08 Best Paper Award, ACM IWCMC'10 Best Paper Award, IEEE Globecom'14 Best Paper Award, IEEE ICC'20 Best Paper Award, IEEE TAOS Technical Committee'20 Best Paper Award, and the CIEIT Best Paper Awards in 2018 and in 2019. Also, he has received IEEE ComSoc Excellent Editor Award for IEEE Transactions on Wireless Communications in 2009. His main research interests include B5G technology in wireless communications such as MIMO, OFDMA, Network coding, Network information theory, Machine learning and Big data analysis.

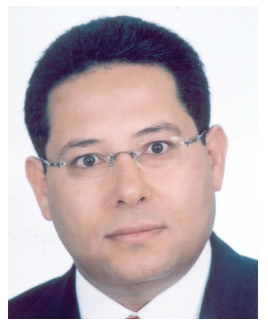

Khaled Ben Letaief (Fellow, IEEE) received the B.S. degree in electrical engineering from Purdue University, West Lafayette, IN, USA, in December 1984, and the M.S. and Ph.D. degrees in electrical engineering from Purdue University, West Lafayette, IN, USA, in Aug. 1986 and May 1990, respectively.

From 1990 to 1993, he was a Faculty Member at the University of Melbourne, Australia. Since 1993, he has been with the Hong Kong University of Science and Technology (HKUST). While at HKUST, he has held many administrative positions, including the Head of the Electronic and Computer Engineering Department, the Director of the Wireless IC Design Center, the founding Director of the Huawei Innovation Laboratory, and the Director of the Hong Kong Telecom Institute of Information Technology. While at HKUST, he has also served as a Chair Professor and the Dean of Engineering. Under his leadership, the School of Engineering has not only transformed its education and scope and produced very high caliber scholarship, it has also actively pursued knowledge transfer and societal engagement in broad contexts. It has also dazzled in international rankings (rising from \#26 in 2009 to \#14 in the world in 2015 according to QS World University Rankings.) From September 2015 to March 2018, he joined HBKU as Provost to help establish a research-intensive university in Qatar in partnership with strategic partners that include Northwestern University, Carnegie Mellon University, Cornell, and Texas A\&M.

Dr. Letaief is a member of the United States National Academy of Engineering, a Fellow of the Hong Kong Institution of Engineers, and a member of the Hong Kong Academy of Engineering Sciences. He is also recognized by Thomson Reuters as an ISI Highly Cited Researcher and was listed among the 2020 top 30 of AI 2000 Internet of Things Most Influential Scholars. He is currently an Internationally Recognized Leader in wireless communications and networks with research interest in artificial intelligence, big data analytics systems, mobile cloud and edge computing, tactile Internet, $5 \mathrm{G}$ systems, and beyond. In these areas, he has more than 630 articles with more than 38350 citations and an H-index of 87 along with 15 patents, including 11 U.S. inventions

Dr. Letaief is the recipient of many distinguished awards and honors including, the 2019 Distinguished Research Excellence Award by HKUST School of Engineering (Highest Research Award and only one recipient/three years is honored for his/her contributions), the 2019 IEEE Communications Society and Information Theory Society Joint Paper Award, the 2018 IEEE Signal Processing Society Young Author Best Paper Award, the 2017 IEEE Cognitive Networks Technical Committee Publication Award, the 2016 IEEE Signal Processing Society Young Author Best Paper Award, the 2016 IEEE Marconi Prize Paper Award in Wireless Communications, the 2011 IEEE Wireless Communications Technical Committee Recognition Award, the 2011 IEEE Communications Society Harold Sobol Award, the 2010 Purdue University Outstanding Electrical and Computer Engineer Award, the 2009 IEEE Marconi Prize Award in Wireless Communications, the 2007 IEEE Communications Society Joseph LoCicero Publications Exemplary Award, and more than 16 IEEE Best Paper awards.

He served as consultants for different organizations, including Huawei, ASTRI, ZTE, Nortel, PricewaterhouseCoopers, and Motorola. He is the founding Editor-in-Chief of the prestigious IEEE TRANSACTIONS ON WIRELESS COMMUNICATIONS and has served on the Editorial Board of other premier journals including, the IEEE JOURNAL ON SELECTED AREAS IN COMMUNICATIONS Wireless Series (as the Editor-in- Chief). He has also been involved in organizing many flagship international conferences. He also served as the President of the IEEE Communications Society from 2018 to 2019, the world's leading organization for communications professionals with headquarter in New York City and members in 162 countries. He is well recognized for his dedicated service to professional societies and IEEE, where he has served in many leadership positions. These include Treasurer of the IEEE Communications Society, the IEEE Communications Society Vice-President for Conferences, the Chair of IEEE Committee on Wireless Communications, a elected member of IEEE Product Services and Publications Board, and the IEEE Communications Society Vice-President for Technical Activities. 